ARTICLE

https://doi.org/10.1038/s41467-018-07823-5

\title{
High-resolution acoustophoretic 3D cell patterning to construct functional collateral cylindroids for ischemia therapy
}

\author{
Byungjun Kang ${ }^{1}$, Jisoo Shin², Hyun-Ji Park (iD ${ }^{2}$, Chanryeol Rhyou', Donyoung Kang ${ }^{1}$, Shin-Jeong Lee ${ }^{3,4}$, \\ Young-sup Yoon ${ }^{3,4}$, Seung-Woo Cho ${ }^{2,5}$ \& Hyungsuk Lee ${ }^{1}$
}

The fabrication of functional tissues is essential for clinical applications such as disease treatment and drug discovery. Recent studies have revealed that the mechanical environments of tissues, determined by geometric cell patterns, material composition, or mechanical properties, play critical roles in ensuring proper tissue function. Here, we propose an acoustophoretic technique using surface acoustic waves to fabricate therapeutic vascular tissue containing a three-dimensional collateral distribution of vessels. Co-aligned human umbilical vein endothelial cells and human adipose stem cells that are arranged in a biodegradable catechol-conjugated hyaluronic acid hydrogel exhibit enhanced cell-cell contacts, gene expression, and secretion of angiogenic and anti-inflammatory paracrine factors. The therapeutic effects of the fabricated vessel constructs are demonstrated in experiments using an ischemia mouse model by exhibiting the remarkable recovery of damaged tissue. Our study can be referenced to fabricate various types of artificial tissues that mimic the original functions as well as structures.

\footnotetext{
${ }^{1}$ School of Mechanical Engineering, Yonsei University, Seoul 03722, Korea. ${ }^{2}$ Department of Biotechnology, Yonsei University, Seoul 03722, Korea. ${ }^{3}$ Division of Cardiology, Department of Medicine, Emory University School of Medicine, Atlanta, GA 30322, USA. ${ }^{4}$ Severance Biomedical Science Institute, Yonsei University College of Medicine, Seoul 03722, Korea. ${ }^{5}$ Center for Nanomedicine, Institute for Basic Science (IBS), Seoul 03722, Korea. These authors contributed equally: Byungjun Kang, Jisoo Shin. Correspondence and requests for materials should be addressed to

S.-W.C. (email: seungwoocho@yonsei.ac.kr) or to H.L. (email: hyungsuk@yonsei.ac.kr)
} 
$\mathrm{n}$ vitro fabrication of functional three-dimensional (3D) tissue is technically challenging but essential for the repair or replacement of impaired tissue in the fields of tissue engineering and regenerative medicine ${ }^{1}$. Many groups have attempted to produce artificial tissues under in vivo conditions involving the co-culture of different types of cells and regulation of growth factors $^{2}$. Recent biomimetic studies have demonstrated that not only the biological and biochemical environments but also the mechanical attributes, including physical and structural properties, of tissues are critical for differentiation, organogenesis, and the maturation of tissue constructs ${ }^{3}$.

Blood vessel organization is required for the efficient growth and function of tissues ${ }^{4}$. Although various methods have been proposed $^{5-9}$, reproducing a blood vessel structure that is complex and multiscale, ranging from micrometers to centimeters, remains difficult ${ }^{4}$. Attempts to mimic artery-like structures using scaffolds or cell sheets ${ }^{8}$ have revealed limitations on the fabrication of microvessels smaller than $50 \mu \mathrm{m}$ in diameter ${ }^{7}$, which have important roles in capillary exchange. In a living organism, microvessels in skeletal muscles have well-aligned cellular and extracellular structures with major paths and branches ${ }^{10}$. For example, the main vessels of the limb skeletal muscle develop collaterally in $3 \mathrm{D}$ to enable efficient and adequate perfusion to the distal leg and foot ${ }^{11}$. In the microvessels, endothelial cells (ECs) and other cells such as pericytes are arranged in high proximity to form blood conduits ${ }^{12}$. The capillary density in human skeletal muscles is in the range of 100-1000 capillaries per $\mathrm{mm}^{2} 13,14$. A distance between capillaries is estimated to be $\sim 30-100 \mu \mathrm{m}$, which is advantageous for diffusion ${ }^{15}$. Co-culture of endothelial and stromal cells promoted the formation of homogeneous microvessels by inducing the self-organization of capillaries ${ }^{6,7,9}$. However, this technique was limited in its ability to regulate the orientation and local distribution of vessels in the vascular tissue ${ }^{7}$. Three-dimensional templating 5,7 and direct cell printing techniques $^{4,6}$ are advantageous for producing geometrycontrolled vasculatures. However, the disadvantages of these approaches include applicable biomaterials, minimum vessel size, vessel area density, and fabrication time $e^{4,6,7}$. To overcome the limitations of existing methods and fabricate vasculatures for disease treatment, it is necessary to develop a technique that comprehensively meets the following requirements: (i) $3 \mathrm{D}$ cellular arrangement akin to native tissue ${ }^{5}$, (ii) extracellular matrix (ECM) environment ${ }^{3}$ with clinically relevant size $^{16}$, (iii) coculture of multiple cell types ${ }^{17}$, (iv) integrated cell-cell junctions $^{18}$, and (v) composed of biocompatible, biodegradable ${ }^{19}$, and tissue-adhesive $\mathrm{e}^{20}$ biomaterials.

Recent studies have shown that pressure fields formed by standing surface acoustic waves (SSAWs) are capable of manipulating microparticles at a high resolution in a noninvasive manner ${ }^{21-26}$. SSAW techniques also exhibit the potential to selectively manipulate various types of microparticles ${ }^{21}$, regulate cell-cell distances ${ }^{22}$, and engineer cellular aggregates such as spheroids $^{25}$. Such high-resolution cell engineering is essential to replicate complex and highly ordered tissues in vivo because obtaining such tissues by current methods, including bioprinting, is difficult. In this study, we introduce a tissue fabrication method by developing a cell patterning technique in a 3D hydrogel matrix using SSAW. Our method is designed to produce an implantable tissue that exhibits physiologically relevant mechanical properties, cellular density and organization. Adipose-derived stem cells and endothelial cells are co-aligned into collateral cylindroids in a biocompatible, biodegradable, and tissue-adhesive catechol-conjugated hyaluronic acid (HA-CA) hydrogel. Enhanced gene expression and growth factor secretion by the tissue fabricated by cell patterning are assessed. The therapeutic potential of $3 \mathrm{D}$ patterned collateral microvessels is tested by performing in vivo implantation using a mouse model of critical limb ischemia. Our methods and results can be applied to fabricate various types of functional tissue constructs mimicking native tissue with improved regenerative efficacy.

\section{Results}

Fabrication of vascular tissue for ischemia therapy. To replicate the structure of the aligned vasculatures in skeletal muscles (Fig. 1a), our acoustophoretic fabrication system was designed to arrange cells into collateral cylindrical patterns at intervals similar to the inter-capillary distance of human skeletal muscle in a 3D hydrogel matrix. To maximize the therapeutic effect of the fabricated tissue with collateral cylindroids in the mouse ischemia model, the size of the hydrogel matrix was determined to be similar to that of a mouse hindlimb muscle.

The vasculatures in the skeletal muscle primarily consist of ECs and mural cells such as pericytes or vascular smooth muscle cells (vSMCs). However, those primary mural cells requiring blood vessel biopsy are not readily accessible cell sources in clinical setting ${ }^{27}$. Adult stem cells exhibit angiogenic effects leading to vascular regeneration. In particular, human adipose-derived stem cell (hADSC) can be acquired in large numbers from human adipose tissue and easily expanded, which is more adequate for clinical approach. More importantly, over last few decades, ADSC has been highlighted as a highly functional therapeutic cell source for neovascularization because of its superior paracrine ability to secrete various angiogenic factors ${ }^{28}$. Previous studies have revealed that ADSCs have the potential to differentiate into pericyte- or vSMC-like cells ${ }^{29,30}$. Therefore, hADSCs were copatterned with human vascular ECs to improve vessel formation and maturation through paracrine signaling ${ }^{29,31}$. Those cells were assembled to form cell-cell junctions at the pressure nodes of the standing pressure field ${ }^{22}$. For the hydrogel, we chose HA-CA owing to several advantages. HA itself has cellular adhesion motifs to interact with binding receptors existing in some cell types including neural stem cells and mesenchymal stem cells $^{32,33}$. And catechol-medicated crosslinking system of HACA provides biocompatibility and excellent tissue adhesiveness. The biophysical and mechanical properties of HA-CA are readily adjustable for various applications, as tested previously by our group $^{34,35}$.

The acoustophoretic fabrication system consisted of a detachable superstrate and the underlying piezoelectric substrate. The superstrate composed of a coverglass, and a polydimethylsiloxane (PDMS) chamber was placed on the substrate. The gap between the superstrate and substrate was filled with a coupling liquid. HA-CA solution mixed with cells was injected into the chamber and enclosed by the coverglass (Fig. 1b). The cells in the solution were patterned into collateral cylindroids at defined intervals in the lateral and vertical directions ( $X$ and $Z$ directions), which replicated the architecture of vessels in hindlimb muscle (Supplementary Fig. 1), by exerting surface acoustic waves which were generated from interdigital transducers (IDTs) and propagated into the gel solution through the coupling liquid (Fig. 1c). As the HA-CA conjugate polymerized, the cells were immobilized at the patterned locations. We arranged the cells uniformly across the entire hydrogel at a clinically relevant scale (Supplementary Fig. 2). The superstrate, including the fabricated tissue, was detached from the substrate and incubated for 1 week after removing the top coverglass (Fig. 1d, e, Supplementary Fig. 3a). After removing the PDMS chamber (Supplementary Fig. 3b), the cell-patterned hydrogel was separated from the bottom coverglass (Fig. 1f, Supplementary Fig. 3c) and transferred onto the hindlimb muscle of a mouse ischemia model (Fig. 1f). We used a fine spatula to transfer the $3 \mathrm{D}$ tissue construct from 
a

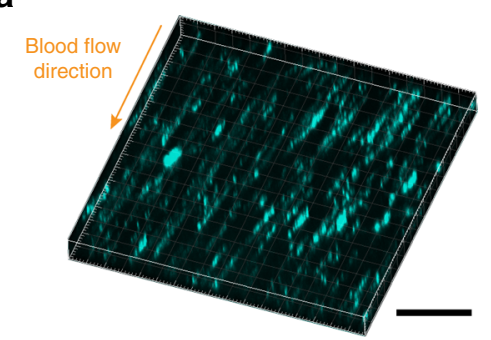

b

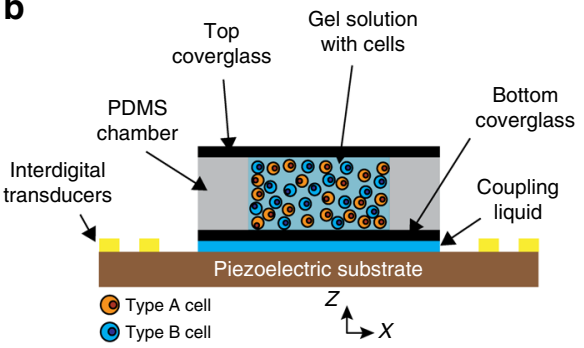

C

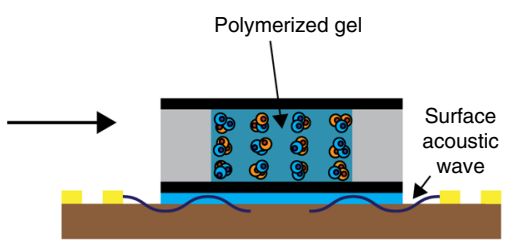

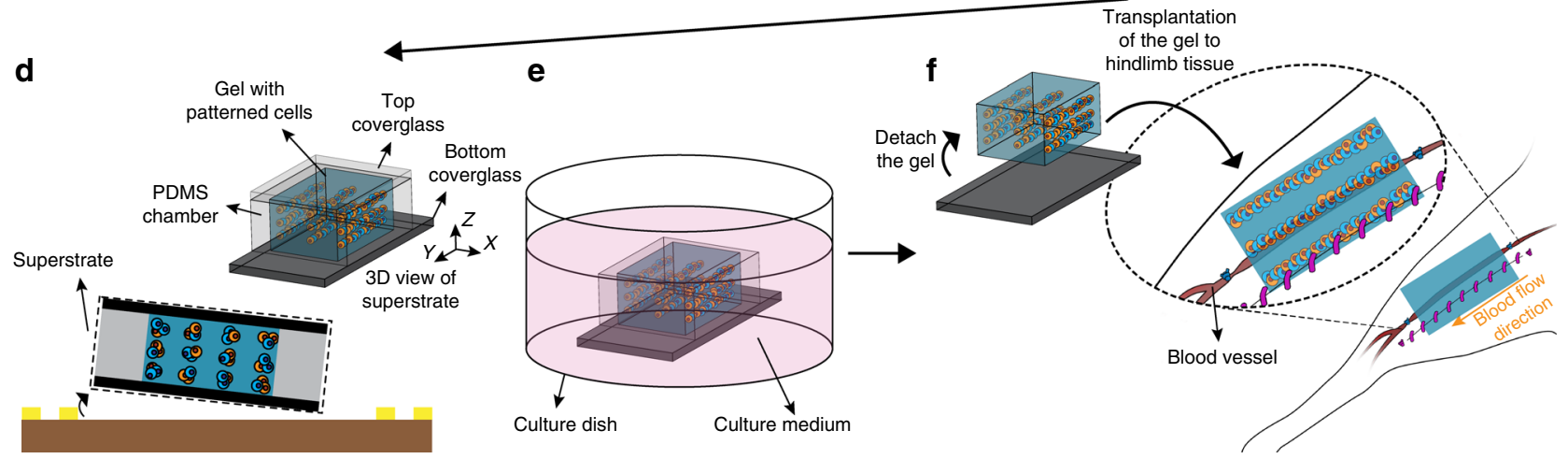

Fig. 1 Fabrication of tissue construct with cells patterned using SSAW for ischemia therapy. a Fluorescent image of 70 kDa FITC-dextran in collateral vessels in mouse hindlimb tissue. Scale bar $=200 \mu \mathrm{m}$. b Injection of HA-CA hydrogel/cell mixture solution into a PDMS chamber on a piezoelectric substrate. $\mathbf{c}$ 3D cell patterning in gel solution using surface acoustic waves. $\mathbf{d}$ Disassembly of the superstrate of the hydrogel with patterned cells from the piezoelectric substrate. e Culturing hydrogel-cell constructs in medium. $\mathbf{f}$ Detachment of hydrogel constructs with patterned cells from the glass and transplantation of the constructs into the mouse hindlimb. The size of construct for in vivo experiments is 6,10, and $0.53 \mathrm{~mm}$ in $X, Y$, and $Z$ directions, respectively. For in vitro experiments, the size of construct is 6,6 , and $0.53 \mathrm{~mm}$ in $X, Y$, and $Z$ directions, respectively

culture substrate to animal model because of difficulty in handling due to its small dimension and low stiffness.

Design of the acoustophoretic tissue fabrication system. A primary component of the acoustophoretic tissue fabrication system is the SSAW-based cell patterning device that enables the cells to be organized into a 3D cylindroid array at high resolution. Briefly, the SSAW is created by the superposition of opposing traveling surface acoustic waves generated by exerting alternating current (AC) electrical signals to IDTs (Fig. 2a). In the coupling liquid above the piezoelectric substrate, a bulk acoustic wave (BAW) is produced perpendicular to the SSAW; this BAW has a displacement amplitude that is largest at the antinodes of the SSAW with intervals that are half of the SSAW wavelength ${ }^{36}$. The generated BAW is transmitted to the hydrogel solution inside the chamber via the coupling liquid and bottom coverglass and is then reflected by the chamber cover. Superposition of the transmitted BAW and its reflection by the chamber cover creates standing pressure fields in the $X Z$-plane (Fig. 2b), resulting in a $3 \mathrm{D}$ acoustic potential. Therefore, cells in the solution can be patterned both horizontally and vertically, with spacing determined mainly by the wavelength of SAW and that of the pressure wave in the gel, respectively 22,37 .

To implement the device, the materials for the cover and the side chamber should be properly chosen. The pattern of the acoustic potential along $Z$ direction became less distinct as decreasing the reflection coefficient of the top cover (Supplementary Fig. 4a). Consequently, when PDMS (intensity reflection coefficient $=0.04$ ) was used as the top cover, cells were rarely patterned along the $Z$ direction (Fig. 2c, d). When a glass (intensity reflection coefficient $=0.56$ ) was used, the cells were arranged in both $X$ and $Z$ directions more clearly (Fig. 2c, d). Additionally, the wave derived from the bottom coverglass can transmit into the top cover through the side chamber. The wave transmitted into the top cover may be reflected at the cover/air interface and propagate into the solution, disturbing the acoustic field in the solution (Supplementary Fig. 4b). The effect of the wave from the side chamber is minimized by selecting a material with a high attenuation coefficient (Supplementary Fig. 4b). Thus, PDMS was chosen as the side chamber material due to its high attenuation coefficient and ease of fabrication.

Successful regenerative medicine involving the transplantation of artificial tissues requires not only optimization of the tissue fabrication system but also the proper selection of biomaterials. Our system was applied to various ECM hydrogels with different crosslinking mechanisms (e.g., collagen, HA-CA, and methacrylate-conjugated HA (HA-MA)) (Supplementary Fig. 5a). In this study, we chose HA-CA hydrogel due to the viability and functionality of the embedded cells, its ability to maintain a cellular arrangement, biodegradability, tissue adhesiveness, and therapeutic ability to repair tissue defects as reported in the liver and hindlimb $b^{34,35}$. Cells subjected to an acoustic field experience not only an acoustic radiation force but also a drag force produced by the viscosity of the medium. As the viscosity increases during polymerization of the HA-CA hydrogel through oxidative crosslinking chemistry, SSAW is applied to the hydrogel-cell mixture with oxidant immediately after being transferred to a chamber.

The concentration of the HA-CA hydrogel was determined by taking the rheological properties of the gel and the alignment degree of patterned cells into account. To maintain the cell arrangement as patterned, a higher concentration was advantageous because the storage modulus of the solidified HA-CA hydrogel increased with the concentration (Fig. 2e, Supplementary Fig. 5b). However, as the concentration increased, it became difficult to manipulate the cells to make the patterns due to the reduced gelation time and the increased complex viscosity of the 
a

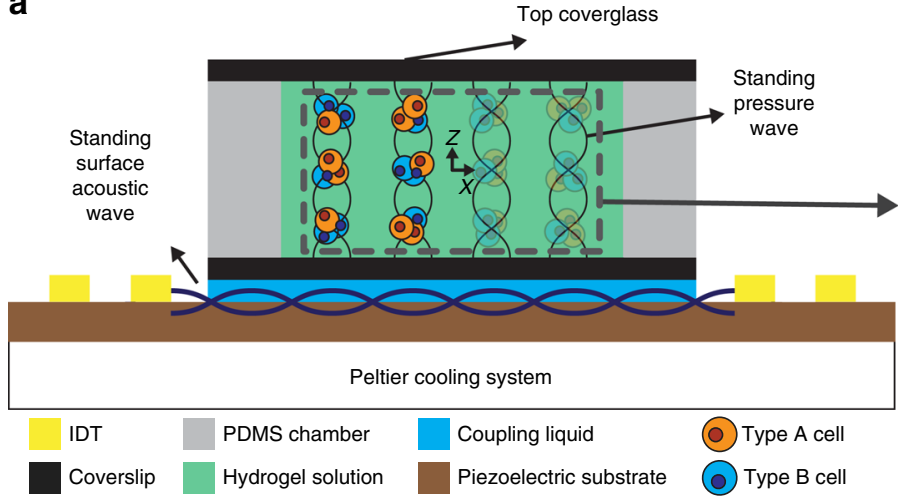

b

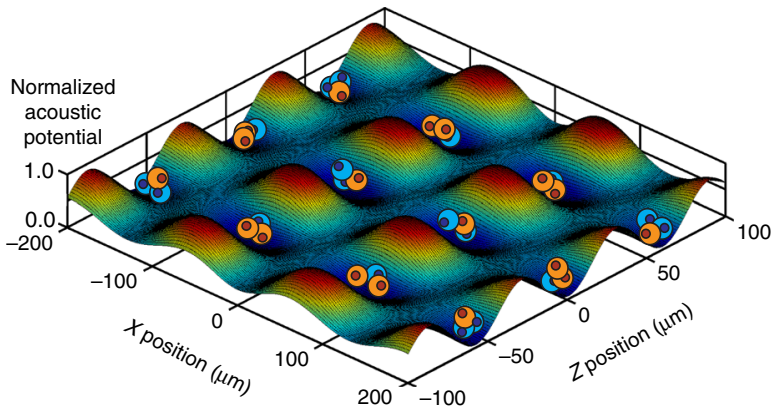

C
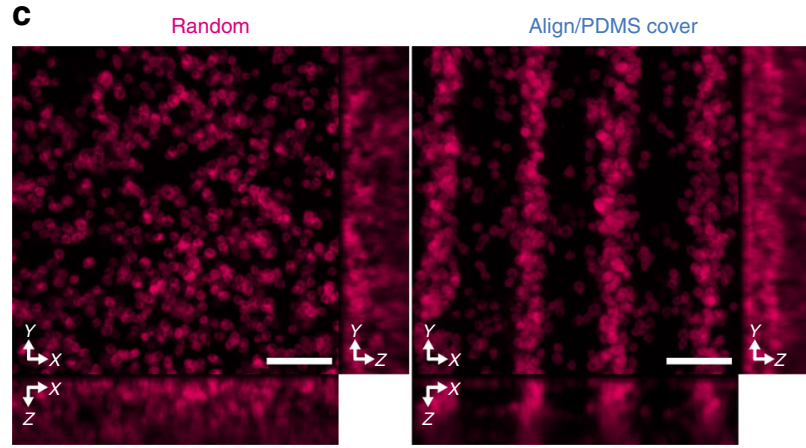

f

e

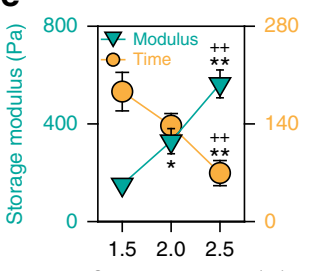

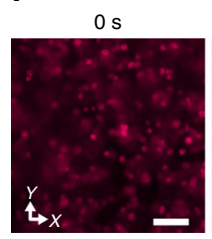

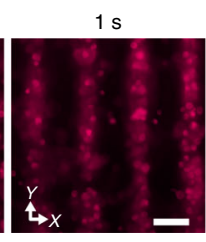

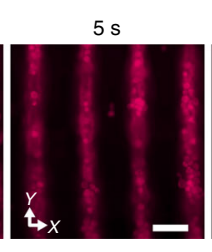

Align/glass cover

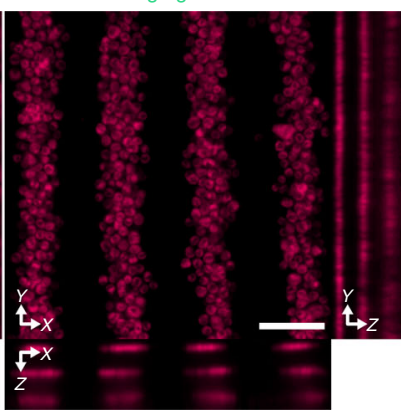

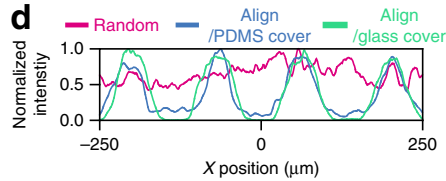
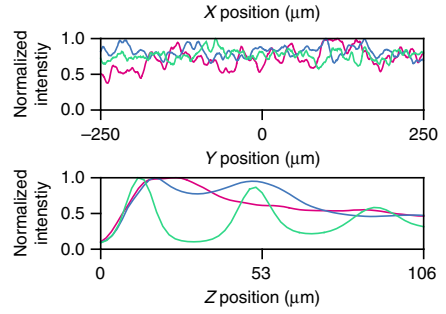

$Z$ position $(\mu \mathrm{m})$

HA-CA concentration (\%)
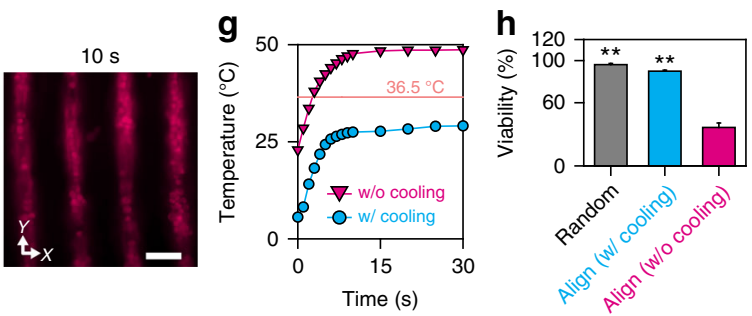

Fig. 2 Acoustophoretic tissue fabrication system. a SSAW system consisting of a piezoelectric substrate, a PDMS chamber, a Peltier cooling system, and two glass coverslips designed to create a pattern of multi-type cells in a 3D hydrogel matrix. $\mathbf{b}$ 3D plot of the acoustic potential field normalized by the maximum value in the center region of the solution. $\mathbf{c} X Y, Y Z$, and $X Z$ projection images from confocal micrographs of Dil-stained cells in hydrogels from the Random group, Align/PDMS cover group and Align/glass cover group. Scale bars $=100 \mu \mathrm{m}$. $\mathbf{d}$ Normalized fluorescence intensity of cells in the $X, Y$, and $Z$ directions in the gel. e Storage modulus at $1 \mathrm{~Hz}$ (cyan triangle, $n=3$ ) and gelation time (orange circle, $n=4$ ) of $\mathrm{HA}-\mathrm{CA}$ hydrogel at various concentrations (technical repeats $=2,{ }^{*} p<0.05$ and ${ }^{* *} p<0.01$ versus $1.5 \%$ HA-CA group and ${ }^{++} p<0.01$ versus $2.0 \%$ HA-CA group, via one-way ANOVA followed by Tukey's multiple comparisons test). $\mathbf{f}$ Time-lapse images of Dil-stained cells patterned by the SSAW system in 2\% HA-CA hydrogel at an input voltage of $90 \mathrm{mVrms}$. Scale bars $=100 \mu \mathrm{m} . \mathbf{g}$ Change in temperature of $2 \% \mathrm{HA}$-CA hydrogel solution during the application of the SSAW with (blue circle) and without (magenta triangle) the cooling system. $\mathbf{h}$ Cell viability of random and aligned cells in hydrogels prepared with and without the cooling system $\left(n=3-4\right.$, technical repeats $=3,{ }^{\star \star} p<0.01$ versus Align without cooling group, via one-way ANOVA followed by Tukey's multiple comparisons test). Error bars represent one standard deviation

polymerizing hydrogel solution (Fig. 2e, Supplementary Fig. 5c). Thus, we chose $2 \%$ HA-CA as the optimal concentration, with a gelation time of $154.8 \mathrm{~s}$ and an initial complex viscosity of 0.13 $\mathrm{Pa} \cdot \mathrm{s}$. We also found that the mechanical response of $2 \% \mathrm{HA}-\mathrm{CA}$ to applied forces was similar to that of mouse hindlimb muscle tissue in indentation tests (Supplementary Fig. 5d).

In fact, necrotic cell death often occurs in the center of large and dense cellular structure due to diffusional limitation of nutrients and oxygen supply. However, the HA-CA hydrogel is highly permeable to molecules in aqueous solution due to its swelling behavior and microporous structure ${ }^{34}$. The high permeability of HA-CA hydrogel was beneficial to efficient mass transfer of fabricated 3D construct for supply of nutrients/oxygen and removal of metabolic wastes, resulting in maintaining cell viability and minimal necrosis in our experiments.
Controlling the temperature is important when fabricating tissues using the acoustophoretic system because attenuation of the pressure wave in the hydrogel increases the temperature, which can reduce cell viability ${ }^{38}$. We noted that the average temperature of deionized water in the chamber increased by an input voltage applied to the system (Supplementary Fig. 6a). When the input voltage was higher than $60 \mathrm{mV}_{\mathrm{rms}}$, the temperature was higher than $36.5^{\circ} \mathrm{C}$, impacting cell survival. To prevent cell death, our system was equipped with a Peltierbased cooling device (Supplementary Fig. 6b). Under our experimental condition $\left(90 \mathrm{mV}_{\mathrm{rms}}\right)$, the duration of the SSAW application for cell patterning was less than $10 \mathrm{~s}$ which was one order shorter than the gelation time of the 2\% HA-CA hydrogel (Fig. 2e, f). The temperature of the hydrogel was kept below $30^{\circ} \mathrm{C}$ (Fig. 2g, Supplementary Fig. 6c), and the cell viability was around 
a
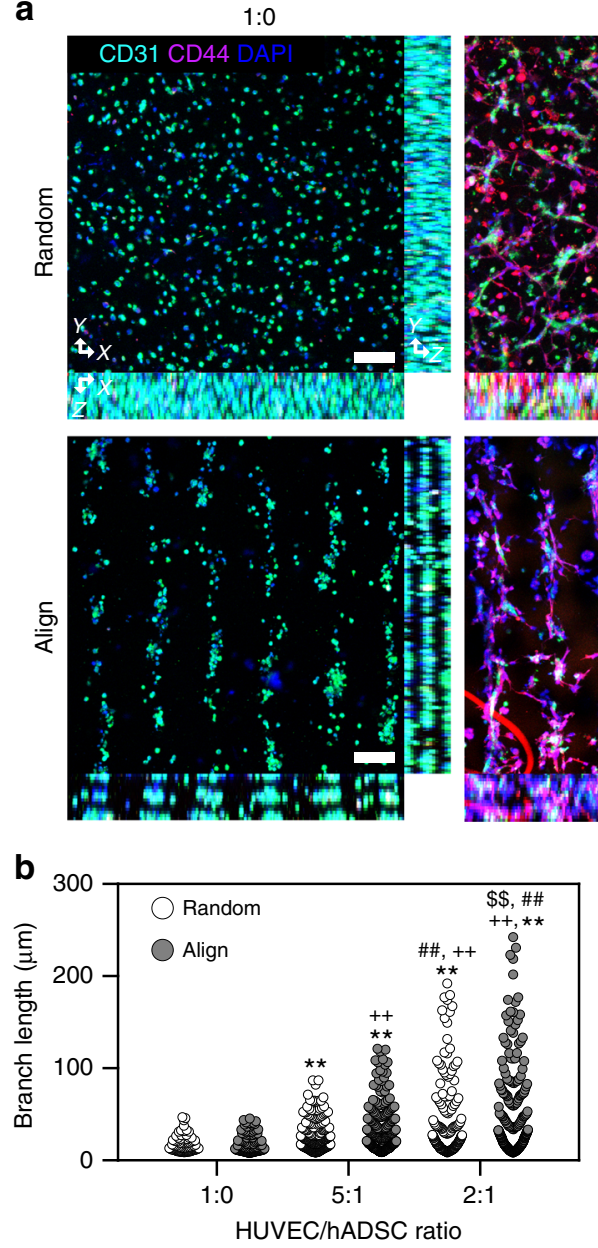

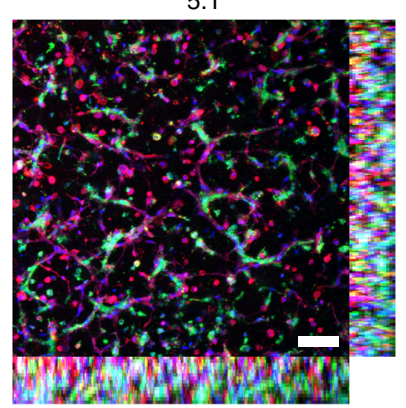

$5: 1$

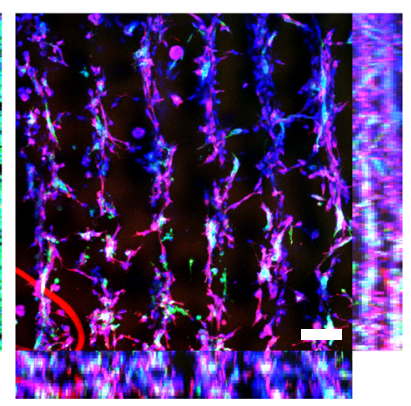

C
2:1
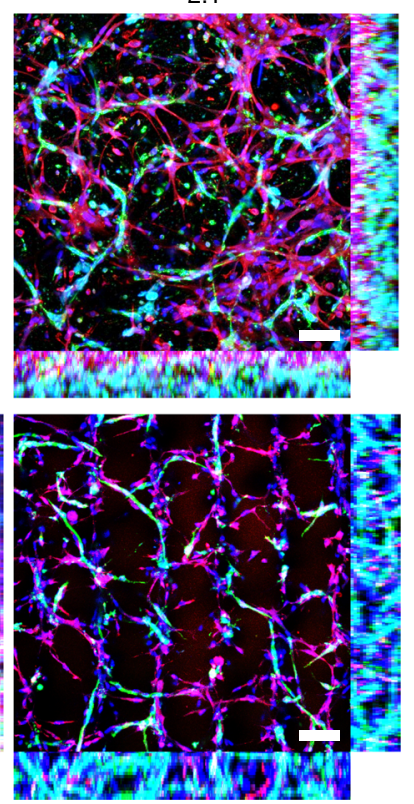
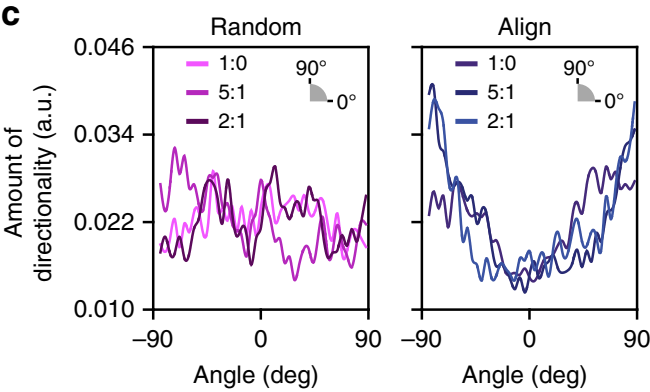

Fig. 3 SSAW-induced 3D cell patterning for the recapitulation of microvessels in hindlimb muscle. a Dual immunofluorescence staining of CD31 for HUVECs and CD44 for hADSCs at various HUVEC/hADSC ratios (1:0, 5:1, 2:1-total cell density: $1 \times 10^{7}$ cells ml-1) 1 week after cultivation (technical repeats $=3$ ). Scale bars $=100 \mu \mathrm{m}$. Fluorescent image-based quantification of $\mathbf{b}$ HUVEC elongation $(n=92-247)$ and $\mathbf{c}$ elongated direction using CD31-stained images (technical repeats $=2,{ }^{\star \star} p<0.01$ versus 1:0 Random group, ${ }^{++} p<0.01$ versus 1:0 Align group, \#\#p<0.01 versus 5:1 Random group, and $\$ p<0.01$ versus 2:1 Random group, via one-way ANOVA followed by Tukey's multiple comparisons test)

90\% (Fig. 2h, Supplementary Fig. 6d). Thus, we were able to fabricate a tissue construct without significant damage to cells using our established system.

A vascular construct replicating vessels in skeletal muscle. Here, we recapitulated $3 \mathrm{D}$ collateral vessel structure in the hindlimb muscle by performing 3D patterning of human stem cells and ECs in lines within an HA-based hydrogel. Without the SSAW application, the cells were randomly distributed within the $3 \mathrm{D}$ hydrogel, showing an alignment index close to the minimum value (Supplementary Fig. 1). In contrast, when SSAW was applied, cells within the HA-CA hydrogel were patterned into lines organizing a collateral vessel-like structure. To obtain a continuous line pattern of cells, we determined the optimal cell density in the 3D hydrogel based on images of a 'Random' group and an 'Align' group. As the cell density increased, the difference between the 'Random' group and the 'Align' group became more significant (Supplementary Fig. 7). When the cell density was higher than $1.0 \times 10^{7} \mathrm{cells} \mathrm{ml}^{-1}$, cells in the 'Align' group formed cylindroids throughout the entire hydrogel area. Given this result, the cell density was optimized as $1.0 \times 10^{7}$ cells $\mathrm{ml}^{-1}$ for in vitro analysis. During angiogenesis, ECs build a vascular network and recruit mural cells to form mature and stable vessels ${ }^{39}$. To fabricate native microvessels with structural and paracrine supports for ECs in the angiogenic process, human umbilical vein endothelial cells (HUVECs) were co-cultured with hADSCs at various ratios (HUVEC:hADSC $=1: 0,5: 1$, or 2:1) 29,31 (Fig. 3a). We maintained the total cell density same, and varied a ratio of HUVEC to ADSC density in experiments. Without hADSCs, the HUVECs maintained a circular morphology and could not extend or sprout at all within the hydrogel until one week after culturing (1:0 group). When the ratio of hADSCs was increased, the HUVECs exhibited a more stretched morphology and sprouted out (5:1 group versus 2:1 group) (Fig. 3a). HUVECs that were co-cultured with hADSCs in the SSAW-induced groups were elongated in a guided direction following a line pattern (Fig. 3b, c).

Improved vascular function and maturation of patterned cells. With respect to vessel maturation, contact and communication between EC-EC and EC-mural cells are critically important for tight junction formation, vascular permeability, and growth factor-dependent EC survival and stabilization ${ }^{18}$. Therefore, we expected the SSAW-mediated alignment of the $3 \mathrm{D}$ vasculature composed of HUVECs and hADSCs to promote the functional maturation of vessels by increasing the local cell density and enhancing cell-cell contacts (Fig. 4a, Supplementary Fig. 7). Enhanced interactions between HUVECs and HUVECs-hADSCs 
a
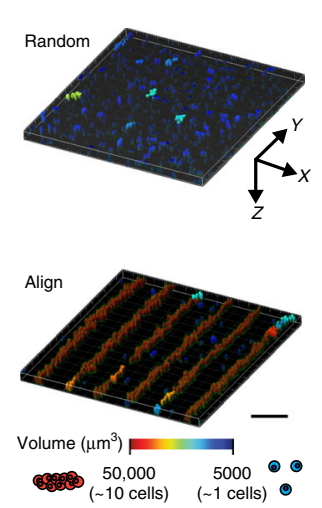

b
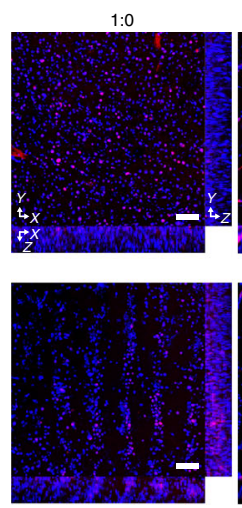

VE-Cadherin DAPI
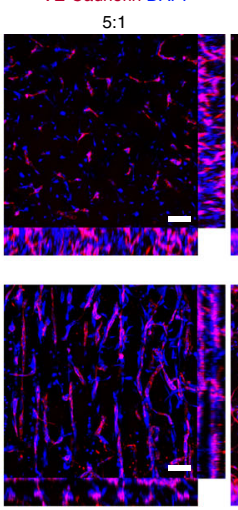

d
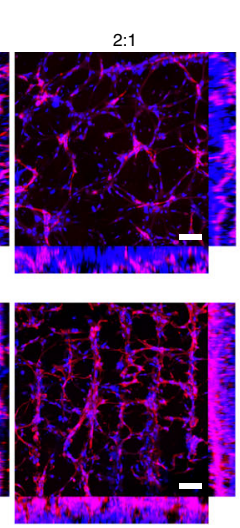
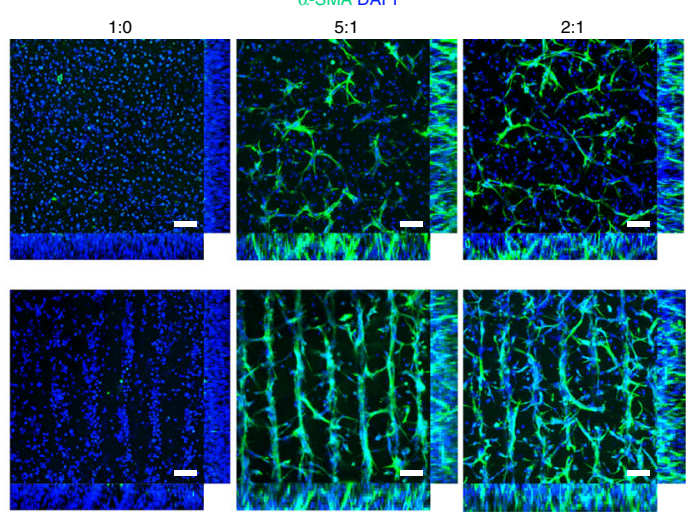

C

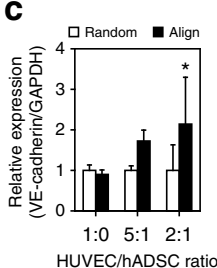

e a Aandom Align

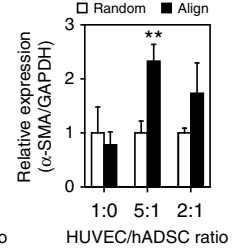

f

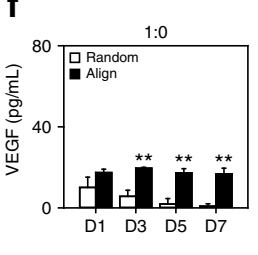

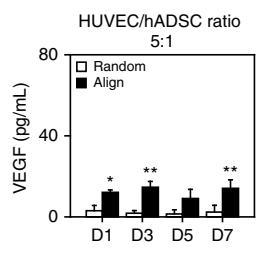

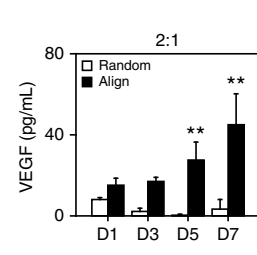

g

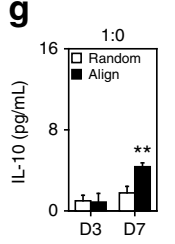

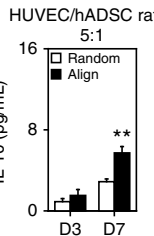

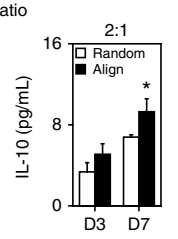

Fig. 4 Effects of enhanced cell-cell contact in the fabricated cell-hydrogel construct on vessel maturation. a Reconstructed 3D images of contacting cells within the hydrogel. Scale bar $=150 \mu \mathrm{m}$. b Representative fluorescent images showing the expression of the EC-specific cell junction marker VE-cadherin at various HUVEC/hADSC ratios (1:0, 5:1, 2:1-total cell density: $1 \times 10^{7}$ cells $\mathrm{ml}^{-1}$ ) (technical repeats $=3$ ). Scale bars $=100 \mu \mathrm{m}$. $\mathbf{c}$ qPCR analysis to quantify the expression of VE-cadherin in aligned/randomly distributed cells within the hydrogel $\left(n=3-6\right.$, technical repeats $=2,{ }^{*} p<0.05$ versus each Random group, via two-way ANOVA followed by Sidak's multiple comparisons test). $\mathbf{d}$ Immunofluorescence staining of cell-hydrogel constructs with the mural cell marker $\alpha$-SMA (technical repeats $=3$ ). Scale bars $=100 \mu \mathrm{m}$. e qPCR analysis to quantify the expression of $\alpha$-SMA in aligned/randomly distributed cells within the hydrogel $\left(n=3-6\right.$, technical repeats $=2,{ }^{\star \star} p<0.01$ versus each random group, via two-way ANOVA followed by Sidak's multiple comparisons test). f ELISA analysis of VEGF secretion by aligned/randomly distributed cells in hydrogels at days $1,3,5$, and 7 ( $n=3$, technical repeats $=2,{ }^{*} p<0.05$ and ${ }^{* *} p<0.01$ versus each Random group, via two-way ANOVA followed by Sidak's multiple comparisons test). $\mathbf{g}$ ELISA analysis of IL-10 secretion by aligned/randomly distributed cells in hydrogels at days 3 and 7 ( $n=3-4$, technical repeats $=2,{ }^{*} p<0.05$ and ${ }^{\star \star} p<0.01$ versus each Random group, via two-way ANOVA followed by Sidak's multiple comparisons test). Error bars represent one standard deviation

induced a significant increase in the expression of vascular endothelial (VE)-cadherin, which is an EC-specific adhesion molecule that has a critical role in vascular maturation ${ }^{40}$ (Fig. $4 \mathrm{~b}$, c). Formation of tight junctions between HUVECs was also observed to be upregulated in 3D-aligned HUVECs with hADSCs as characterized and compared from the immunostained images of Zonula occludens-1 (ZO-1), a marker of tight junctions, indicating the important role of increased cell-cell contacts in formation of functional endothelium with tight junction (Supplementary Fig. 8a, b). Furthermore, the formation of vascular lumen-like structure was observed within 3D-aligned construct at HUVEC/hADSC 2:1 ratio, as shown in ortho-view of magnified images immunostained by ZO-1 (Supplementary Fig. 8c). In addition to upregulated expression of VE-cadherin, we confirmed that increased contact between HUVECs and HUVECs-hADSCs in SSAW-applied groups upregulated gene expression of Tie2 and von Willebrand Factor (vWF), which are highly related to vascular maturation ${ }^{41}$, especially in co-cultured groups at 2:1 ratio of HUVEC/hADSC (Supplementary Fig. 9a). Furthermore, increasing direct cell-cell contact between HUVECs and hADSCs via SSAW-induced 3D alignment upregulated the expression of a mural cell marker-smooth muscle alpha-actin ( $\alpha$-SMA) -in hADSCs (Fig. 4d, e). Thus, a-SMA-expressing hADSCs in 3Dpatterned vascular structures may have a mural cell-like role in microvessel maturation and stabilization ${ }^{42}$.

Furthermore, platelet-derived growth factor (PDGF) secretion of HUVECs, which has a critical role in the maturation of vessel through the recruitment of pericytes ${ }^{43}$, was promoted in SSAWapplied co-cultured groups, due to the enhanced cell-cell interactions (Supplementary Fig. 9b). hADSCs have been regarded as an attractive therapeutic cell source for ischemic diseases due to their paracrine ability ${ }^{44}$. hADSCs have shown immune-modulating effects that attenuate ischemia-induced inflammation by secreting anti-inflammatory factors, in addition to exerting angiogenic effects ${ }^{45}$. Therefore, the levels of angiogenic factors (vascular endothelial growth factor; VEGF) and anti-inflammatory cytokines (interleukin-10; IL-10) secreted from both aligned and randomly distributed cells within HA-CA hydrogels at various HUVEC/hADSC ratios (1:0, 5:1, and 2:1) were compared on days 1, 3, 5, and 7 after encapsulation (Fig. 4f, g). Increased cell-cell contacts in the aligned groups through acoustophoretic patterning resulted in a significantly higher secretion of VEGF and IL-10 compared to that in the random groups, contributing to enhanced angiogenesis and decreased apoptosis at ischemic defect sites. By increasing the number of hADSCs, more extensive secretion of VEGF and IL-10 was detected. Taken together, HUVEC/hADSCs co-cultured at a 2:1 ratio were expected to show the greatest therapeutic potential for angiogenesis and tissue healing when they were aligned and condensed in 3D collateral vessel-like structures within the hydrogel.

To demonstrate the applicability of our methodology further, we fabricated a 3D vascular construct using human induced pluripotent stem cell (hiPSC)-derived EC (hiPSC-EC), instead of HUVEC. hiPSC-ECs were well-aligned with hADSCs (Supplementary Fig. 10a) and they exhibited more branches along the cell alignment compared to random group (Supplementary Fig. 10b, c). This result shows a potential that our technique 
a

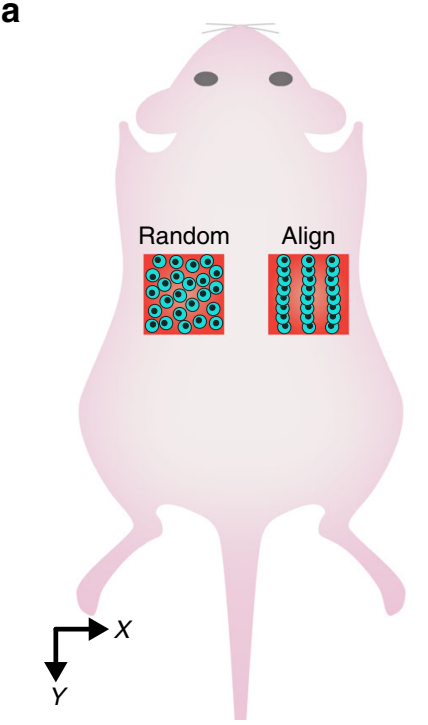

d

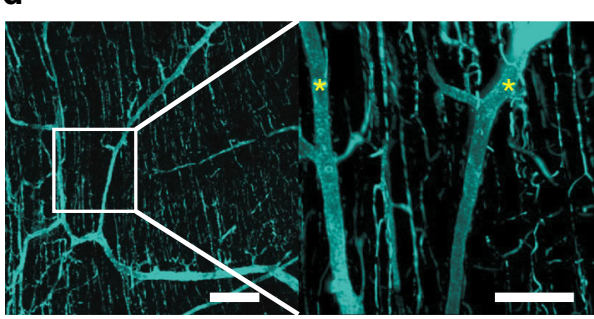

b

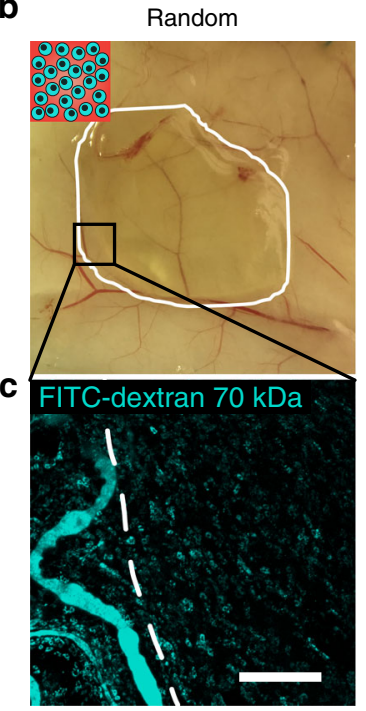

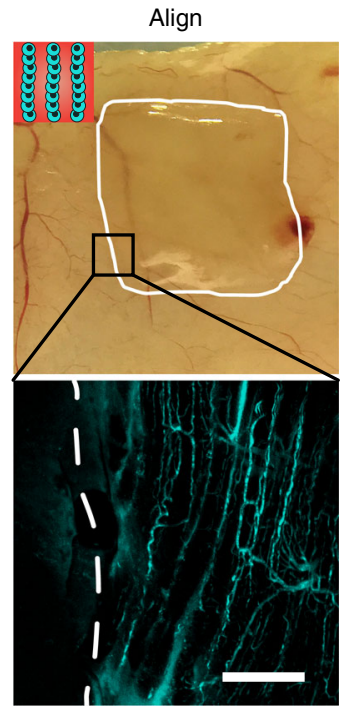

e
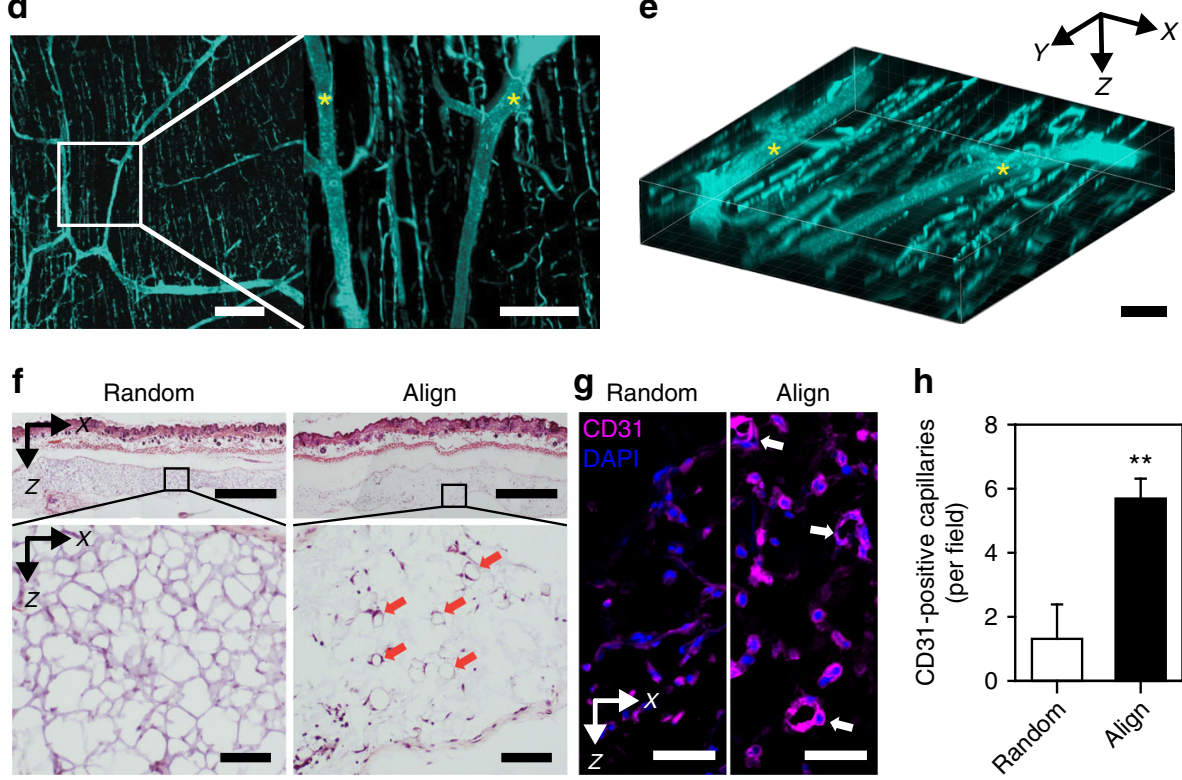

h

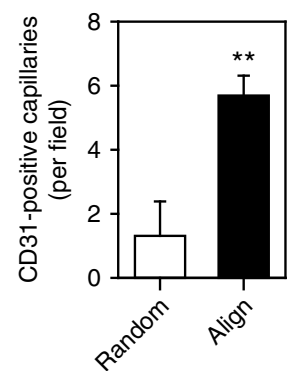

Fig. 5 Integration of patterned microvessels with the host vasculature in transplantation. a Schematic illustration showing the transplantation of randomly distributed and aligned cell-hydrogel constructs into the subcutaneous space of the mouse back. $\mathbf{b}$ Gross view of harvested back-skin tissues with cellhydrogel constructs 1 week after transplantation. c Fluorescent images of $70 \mathrm{kDa}$ FITC-dextran-perfused vessels in one part of the image (b). Scale bars $=$ $200 \mu \mathrm{m}$. Representative d 2D images and e 3D image of FITC-dextran-labeled vessels in transplanted 3D-aligned cell-hydrogel constructs (HUVEC/ hADSC ratio at 2:1-total cell density: $1.2 \times 10^{7}$ cells $\mathrm{ml}^{-1}$ ) 1 week after transplantation. The asterisks indicate infiltrated host vessels. Scale bars $=200 \mu \mathrm{m}$ for the left image of $\mathbf{d}, 100 \mu \mathrm{m}$ for the right image of $\mathbf{d}$, and $50 \mu \mathrm{m}$ for $\mathbf{e} . \mathbf{f} \mathrm{H \& E}$-stained images of cross-sectioned 3D cell-hydrogel constructs with adjacent skin tissue 1 week after transplantation. Red arrows indicate vessel-like structures. Scale bars $=500 \mu \mathrm{m}$ (upper image) and $200 \mu \mathrm{m}$ (lower image). g Immunofluorescence staining of CD31 in a 3D cell-hydrogel construct 1 week after transplantation. White arrows indicate CD31-positive capillaries with lumen structure. Scale bars $=50 \mu \mathrm{m}$. h Quantification of CD31-positive capillary density in a 3D cell-hydrogel construct 1 week after transplantation $\left(n=3\right.$, technical repeats $=2,{ }^{\star \star} p<0.01$ versus Random group, via unpaired $t$-test). Error bars represent one standard deviation

can be applied to a clinical therapy which requires patient-specific autologous cells ${ }^{46}$.

Integration of vascular constructs into host vasculature. To examine the integration of the engineered $3 \mathrm{D}$ vascular construct with aligned HUVECs/hADSCs at a 2:1 ratio into the host vasculature, the constructs were transplanted subcutaneously into the dorsal regions of mice (Fig. 5a). A subcutaneous pocket on the back of the mouse was chosen as the transplantation site because this region is one of the best in vivo sites to examine vascular connections and integration into host vasculature ${ }^{47}$, and transplanted hydrogel constructs can remain intact. Fluorescein isothiocyanate (FITC)-conjugated dextran (FITC-dextran, $70 \mathrm{kDa}$ ) was perfused via tail vein injection at day 7 post-transplantation, and then the transplanted 3D vascular constructs were imaged with the surrounding skin tissue (Fig. 5b-e). Surprisingly, the extensive FITC-dextran perfusion of capillaries was observed only with the $3 \mathrm{D}$ constructs showing parallel lines of cellular alignment within the HA-CA hydrogel (Fig. 5c-e). The host vessels infiltrated into the transplants and further branched into engineered capillaries, which had longitudinal alignment (Fig. 5d, e). 
These results indicated that $3 \mathrm{D}$-patterned alignment in the hydrogel construct promoted the formation of perfusing microvessels under guided direction by facilitating the direct integration of transplanted vessel structures into the native host vasculature. It occurred because aligned ADSCs attracted and guided native blood vessels toward the alignment direction of the transplanted tissue via paracrine secretion of various angiogenic factors ${ }^{48}$. This phenomenon indicates that patterned cells not only mimic the vessel-specific structures, but also have an important role in integrating with native vessels by spatially regulating angiogenic signaling.

Histological analyses of the $3 \mathrm{D}$ vascular construct with adjacent skin tissue revealed aligned microvessel-like structures within the hydrogel network. In contrast to the randomly distributed cellhydrogel construct, the aligned group showed a larger number of vessel-like structures with lumens in the hydrogel network (Fig. 5f). Immunohistochemical staining for CD31 revealed that the observed vessel-like structures in hematoxylin \& eosin (H\&E)-stained images were primarily CD31-positive capillaries (Fig. 5g). As expected, the larger number of CD31-positive capillaries was detected in aligned group $(5.7 \pm 0.8$ capillaries per field, mean \pm one standard deviation) than in random group $(1.3 \pm 1.3$ capillaries per field) (Fig. 5h). At 3 weeks after subcutaneous transplantation, the HA-CA hydrogels with aligned structures degraded more rapidly, whereas the hydrogels with random structures remained fully intact (Supplementary Fig. 11a). These results may indicate that ECM remodeling in the transplanted hydrogel construct is accelerated by a $3 \mathrm{D}$ vascular arrangement that promotes the formation of perfused vessels and host vascular integration. Importantly, at 3 weeks after transplantation, the development of numerous well-aligned collateral microvessels that were connected to host vessels was observed at sites of aligned hydrogel degradation in skin tissue, whereas both vessel formation and host vessel integration were rarely observed at the sites of random hydrogel transplants, and host vessel integration was also not observed (Supplementary Fig. 11b). Taken together, our results clearly showed that 3D-aligned vascular constructs fabricated by SSAW produced geometrically controlled and functional neovascularization that integrated with the host vasculature.

Therapeutic angiogenesis by vascular constructs. Before investigating the therapeutic efficacy of fabricated vascular constructs, we confirmed whether the $3 \mathrm{D}$ alignment of cells in HA-CA hydrogel (2:1 ratio of HUVEC/hADSC) was maintained even after transplantation into ischemic muscle in a mouse model of hindlimb ischemia. Aligned and randomly distributed cellhydrogel constructs were transplanted into ischemic hindlimbs of mice and retrieved at day 3 post-transplantation. Immunohistochemical staining of the retrieved hydrogels for the detection of an hADSC marker (CD44) indicated that the 3D-aligned structure in the transplanted HA-CA hydrogel construct was well-preserved in vivo (Fig. 6a).

Finally, we assessed the therapeutic efficacy of 3D-aligned cellhydrogel constructs formed by SSAW induction by transplanting the constructs, which were pre-matured in vitro for 1 week, into a mouse model of hindlimb ischemia ${ }^{49}$. Twenty-eight days after transplantation, the aligned group showed significantly improved ischemic limb salvage (75\%) with reduced loss of feet and limbs and reduced necrosis (Fig. 6b, c). Post-ischemic blood flow recovery was sequentially analyzed by using a Doppler imaging technique over 4 weeks. At four weeks after transplantation, $72.8 \%$ of blood flow was restored in the aligned group, whereas only $31.6 \%, 31.8 \%$, and $42.0 \%$ recovery of blood perfusion was observed in the no-treatment, hydrogel-alone, and random groups, respectively (Fig. 6b, d). The native microvesselmimicking vascular structure, which contains collaterally aligned ECs and supporting stromal cells and exhibits matured vascularization via enhanced direct cell-cell contact, may contribute to improved blood perfusion and salvage of the ischemic limb. In histological analyses performed 4 weeks after transplantation (Fig. 6e, g), H\&E and Masson's trichrome staining indicated that muscle fiber degeneration and fibrosis in ischemic muscles were significantly prevented in the aligned group compared with other control groups (no-treatment, hydrogel-alone, and random) (Fig. 6e, f) due to the antiinflammatory and angiogenic effects confirmed in in vitro experiments. Neovascularization was evaluated by immunohistochemical staining of CD31-positive capillaries and a-SMApositive arterioles in ischemic muscles (Fig. 6g). The aligned group exhibited a significant increase in the densities of newly formed capillaries and arterioles, and the average size of arterioles was higher than that in the other groups (Fig. 6h). More importantly, immunohistochemical staining of human-specific CD44 for the detection of hADSCs and CD31 for the detection of capillaries indicated that there were larger numbers of blood vessels originating from human cells in the aligned group than in the random group (Fig. 6i, j). These results clearly indicated that the transplantation of 3D-aligned vascular cell-hydrogel constructs generated by SSAW contributed to highly effective functional blood vessel formation, leading to improved therapeutic angiogenesis for the treatment of critical peripheral ischemic diseases.

\section{Discussion}

Our acoustophoretic technique overcomes the limitations of conventional microvessel fabrication techniques in terms of fabrication time, material selection, and cell manipulation resolution $4,6,7$. The duration required for tissue fabrication in our method is similar to the gelation time of the hydrogel because the cell patterning occurs in less than $10 \mathrm{~s}$. In addition, our patterning technique is advantageous for fabricating various types of tissues due to its versatility, as demonstrated in experiments using hydrogels with various crosslinking systems (Supplementary Fig. 5a). The resolution of our technique can be estimated by measuring distances between cell patterns and width of aligned cell patterns. The average of $X$-directional interval was measured to be 64,124 , and $132 \mu \mathrm{m}$ depending on the IDT pattern. These values are very similar to the half wavelength of applied SAW, which were $62.5,125$, and $140 \mu \mathrm{m}$, respectively. The $Z$-directional interval was also varying in the range of $17-37 \mu \mathrm{m}$. However, the ratio of $Z$-directional interval to X-directional one was not significantly varied independent of SAW wavelength (Supplementary Fig. 12a, b). Considering the wavelength of conventional SAW devices 36 , the intervals between cell patterns can be modulated in the rage of few microns to hundreds microns. A width of aligned cell pattern was characterized as a function of cell density. It increased from 25 to $51 \mu \mathrm{m}$ when the cell density

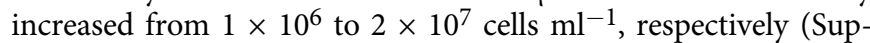
plementary Fig. 12c). Physical contact between cells patterned by our method is ensured due to the attractive secondary Bjerknes forces generated by the scattering of acoustic field ${ }^{50}$. As a result, it can help to enhance the cell-cell communication. The control of cell aggregation was not readily obtained in $3 \mathrm{D}$ printing methods ${ }^{51}$.

In addition to the simple line-shaped cell pattern in this study, it is possible to make more complicated patterns by using IDTs of various shapes. For example, when SSAW is generated by bidirectionally arranged IDTs, cells can exhibit alignments in the lateral, horizontal, and vertical directions ( $X, Y$, and $Z$ directions) 


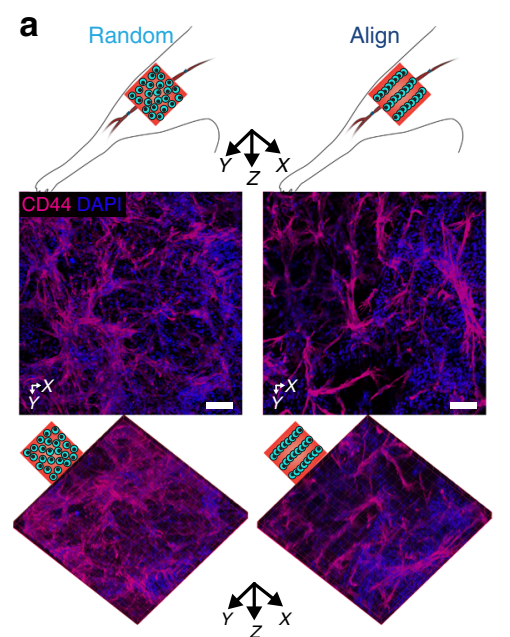

b

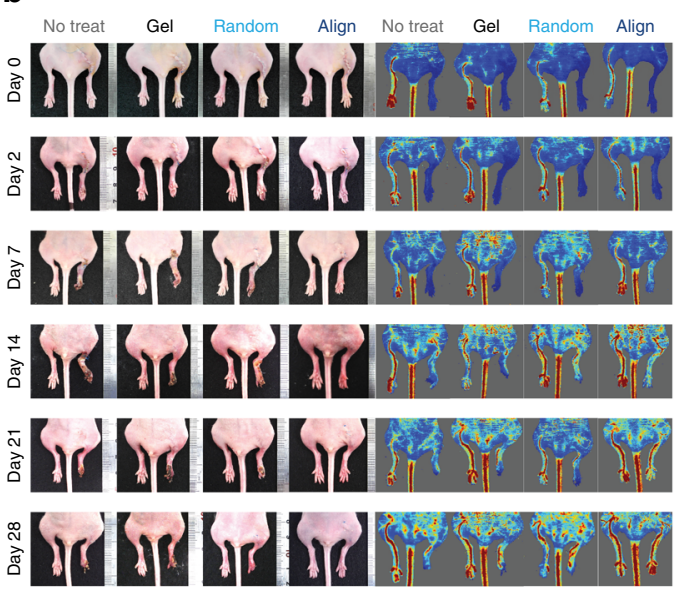

C
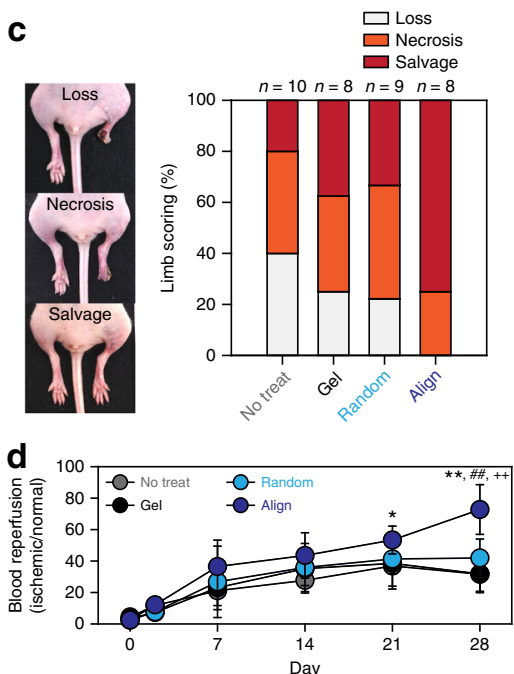

e
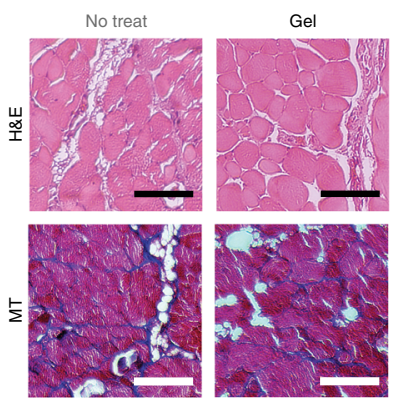

h

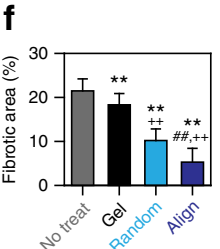

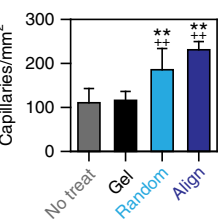
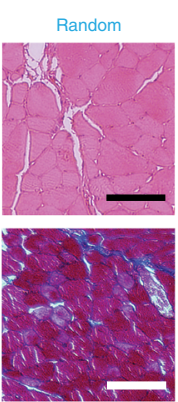

Align
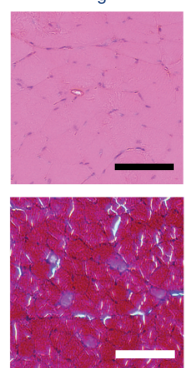

g
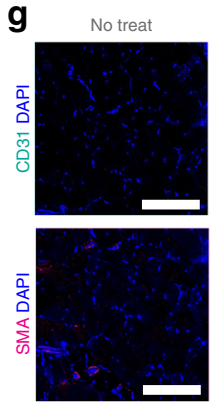

i
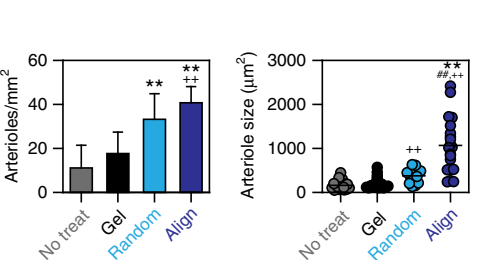
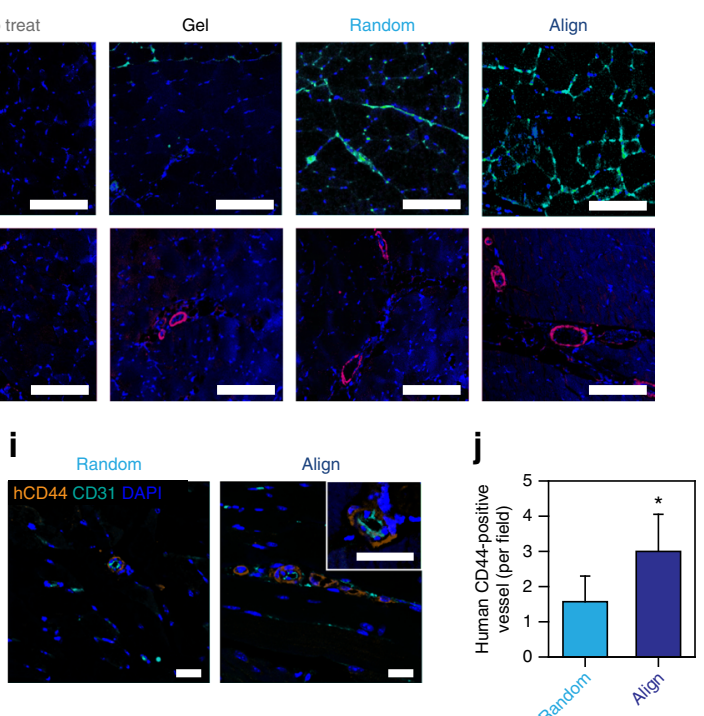

Fig. 6 Enhanced therapeutic angiogenesis via transplantation of the fabricated cell-hydrogel construct. a Immunofluorescence staining of CD44 for determining the maintenance of cell alignment in retrieved cell-hydrogel constructs (HUVEC/hADSC ratio at 2:1-total cell density: $1.2 \times 10^{7}$ cells $\mathrm{ml}^{-1}$ ) 3 days after transplantation into ischemic hindlimb of mouse. Scale bars $=100 \mu \mathrm{m}$. b Representative serial photographs (left) and blood perfusion images (right) of ischemic hindlimb at days $0,2,7,14,21$, and 28 after cell transplantation. c Scoring for the physiological status of ischemic limbs on day 28 . d Quantification of the relative blood perfusion rate of ischemic limb to normal limb in each group $\left(n=7-8\right.$, technical repeats $=2,{ }^{\star} p<0.05$ and ${ }^{\star *} p<0.01$ versus No-treatment group, $++p<0.01$ versus Gel group, and \#\# $<0.01$ versus Random group, via two-way ANOVA followed by Sidak's multiple comparisons test). e H\&E (upper) and Masson's trichrome (lower) staining of the cross-sectioned ischemic muscles 28 days after cell transplantation. Scale bars $=100 \mu \mathrm{m}$. $\mathbf{f}$ Quantification of fibrotic area in the ischemic site $\left(n=10\right.$, technical repeats $=2,{ }^{\star \star} p<0.01$ versus No-treatment group, ${ }^{++} p<0.01$ versus Gel group, ${ }^{\#} p<0.01$ versus Random group, via one-way ANOVA followed by Tukey's multiple comparisons test). $\mathbf{g}$ Immunofluorescence staining of CD31 (capillaries; upper) and $\alpha$-SMA (arterioles; lower) in the cross-sectioned ischemic muscles 28 days after cell transplantation. Scale bars $=100 \mu$ m. h Quantification of CD31-positive capillary density ( $n=7$, left), $\alpha$-SMA-positive arteriole density $(n=7$, middle) and size ( $n=20$, right) in ischemic limb tissue (technical repeats $=2,{ }^{\star \star} p<0.01$ versus No-treatment group, ${ }^{++} p<0.01$ versus Gel group, and \#\#p $<0.01$ versus Random group, via one-way ANOVA followed by Tukey's multiple comparisons test). i Dual immunofluorescence staining with human-specific anti-CD44 (for transplanted hADSCs) and mouse/human-co-reactive anti-CD31 (capillaries) in the cross-sectioned ischemic regions 28 days after cell transplantation. Scale bars $=20 \mu \mathrm{m}$. j Quantification of human CD44-positive vessels per field in ischemic limb tissue $\left(n=7-10\right.$, technical repeats $=2,{ }^{*} p<0.05$ versus Random group, via unpaired $t$-test). Error bars represent one standard deviation

(Supplementary Fig. 12d). When SSAW is combined with other techniques such as the frequency modulation using chirped $\mathrm{IDTs}^{52}$ or the phase modulation ${ }^{21}$, it can be possible to arrange different types of microparticles or cells at distinct locations (Supplementary Fig. 13). In conclusion, our method can be applied to fabricate various tissues with more complicated organization, such as neuro-muscular and epithelial-endothelial junctions.
Two approaches for therapeutic transplantation for ischemia have been suggested: stem cells $s^{6,7,9}$ and pre-aligned microvessel constructs $^{5}$. In the former, angiogenesis and tissue recovery are promoted by paracrine effects and vascular differentiation. However, formation of the vessel network requires weeks, and the structures of the vessels are random and immature ${ }^{6,7,9}$. The microvessels formed by stem cell therapy are often unstable and do not persist in the long term ${ }^{53,54}$. The aligned microvessels 
constructed by using a 3D template or direct cell printing ${ }^{55}$ help form functional vessels in murine ischemia, leading to a rapid recovery ${ }^{5}$. Mirabella et al. ${ }^{5}$ demonstrated the successful therapeutic application of $3 \mathrm{D}$-printed vascular constructs with aligned ECs for treatment of hindlimb ischemia and myocardial infarction. However, the paracrine effects mediated by the EC constructs may not be sufficient for angiogenesis and vascular maturation. Also, the vessel area density, defined as the number of vessels in a unit area of cross section, was slightly smaller than that observed in native tissue in vivo ${ }^{56}$. The resolution that determines the spatial distribution of vessels may also need further improvement.

Our method is complementary, and it is able to arrange stem cells and ECs in parallel lines simultaneously. Patterning cells in both the lateral and vertical directions increased the area density of microvessels and further enhanced the paracrine effect. This effect, encompassing VEGF and IL-10 secretion induced by HUVEC/hADSC co-culture, was increased by SSAW-induced cell aggregation, resulting in the significant enhancement of angiogenesis and tissue recovery. In addition, improvement of vascular stability and maturation by patterned HUVEC/hADSC co-culture induced the integration of the $3 \mathrm{D}$ microvessel networks into the host vasculature and produced perfusable microvessels. Due to the effective transport of blood to the end of the leg through the large arterioles and microvessels produced at the centimeter scale 5,57 , the transplanted tissue constructs exhibited a significant increase in the rate of tissue recovery in the ischemic model. Nonetheless, our SSAW-based 3D patterning will be further improved by future studies investigating (i) the biophysical effects of cylindrical cell arrangement and inter-cylindroid spacing on cell maturation and vascular generation and (ii) the mechanism of vascular integration between implanted vessels and host vessels in vivo. We expect that our therapeutic $3 \mathrm{D}$ vascular tissue will be effective not only for the treatment of human vascular diseases but also other diseases such as organ malperfusion ${ }^{5}$.

Each organ in the body has its own vasculature with distinctive functions ${ }^{15,58}$. Common and unique functions of the vasculature in each organ are accomplished by organ-specifically differentiated ECs ${ }^{15,58}$. Therefore, the tissue-specific structure of ECs should be taken into account in producing the organotypic vasculature using the acoustophoreic cell patterning system. Also, the lymphatic vascular systems are closely interconnected with blood vascular system ${ }^{59}$. It is essential for maintaining tissue fluid homeostasis, fat absorption and immunological surveillance ${ }^{59}$. In order to fabricate a more physiologically relevant vascular tissue, it is probably required to engineer both blood and lymphatic vessels in one tissue construct.

In conclusion, we developed a SSAW-based 3D cell-patterning technique to fabricate a therapeutic tissue consisting of aligned microvessels organized at a high resolution. The fabricated tissue exhibited the upregulated expression of cell-cell junctions and mural cell markers and the increased secretion of angiogenic factors and anti-inflammatory cytokines. The transplanted 3D vascular construct formed perfusable and aligned vessels in mouse models and showed therapeutic angiogenesis in a hindlimb ischemia mouse model. Our study describes an innovative method - a 3D acoustophoretic cell patterning technique utilizing a biocompatible hydrogel - to engineer functional tissues exhibiting in vivo-like structural features and regenerative efficacy for therapeutic applications and drug testing.

\section{Methods}

Preparation of the SSAW device. The SSAW device was composed of a piezoelectric substrate and IDTs. Two sets of IDTs consisting of a 2 - $\mu \mathrm{m}$-thick aluminum layer were deposited and patterned on a $500-\mu \mathrm{m}$-thick $128^{\circ} \mathrm{Y}$-cut lithium niobate wafer by a conventional photolithography technique ${ }^{22}$. The width of the IDTs and the interval between each IDT finger were $70 \mu \mathrm{m}$. Each IDT set in the SSAW device was connected to an arbitrary waveform generator (33622A, Keysight Technologies, Santa Rosa, CA, USA) and a radio frequency (RF) amplifier (LZY-22+, MiniCircuits, Brooklyn, NY, USA). The wavelength of the surface acoustic wave was $280 \mu \mathrm{m}$. The frequency of the SAW was $13.928 \mathrm{MHz}$.

Superstrate preparation. Our system was designed to handle a fabricated tissue construct with ease for transplantation by allowing detachment of a superstrate containing the tissue from the substrate ${ }^{26}$. The detachable superstrates consisted of a coverglass $(10.5 \times 22 \mathrm{~mm})$ and a PDMS chamber. To fabricate the detachable superstrates, PDMS solution (Sylgard 184, Dow Corning, Midland, MI, USA) with a 10:1 (mass ratio of base to curing agent) mixing ratio was first poured onto an acryl rectangular block $(100 \times 100 \times 10 \mathrm{~mm})$ with 0.53 -mm-thick spacers and degassed in a vacuum pump for $1 \mathrm{~h}$. Another acryl block was then placed on the solution and baked at $60^{\circ} \mathrm{C}$ for $24 \mathrm{~h}$. A chamber was prepared by cutting the PDMS block using a customized cutter. After cleaning and wetting the chamber using alcohol, the chamber was placed in the center of the coverglass $(10.5 \times 22 \mathrm{~mm})$. By drying the PDMS/glass superstrate at $60^{\circ} \mathrm{C}$ for $30 \mathrm{~min}$, the PDMS chamber was reversibly bonded to the bottom glass by Van der Waals interactions. This allowed us to disassemble the superstrate containing the fabricated tissue for transplantation. The superstrate was sterilized under UV illumination for more than $1 \mathrm{~h}$ prior to the experiment. The size of the PDMS chamber was $6 \times 6 \times 0.53 \mathrm{~mm}$ for in vitro experiments and $6 \times 10 \times 0.53 \mathrm{~mm}$ for in vivo experiments. The chamber in the superstrate was covered by another coverslip.

Preparation of a Peltier-based cooling system. The SSAW device was bonded to a flat aluminum chip using conductive epoxy to minimize the effect of bulk acoustic wave inside the piezoelectric substrate ${ }^{60}$. The aluminum chip was thermally coupled with a Peltier cooling system using a silicone-based thermal pad (Arctic, Braunschweig, Niedersachsen, Germany). The Peltier cooling system was composed of aluminum plate, Peltier element, heat sink, fan, temperature controller, and temperature sensor. A temperature sensor was attached to the aluminum plate, and the temperature was controlled by the proportional-integralderivate (PID) method. The temperature change in the solution was measured by a thermocouple probe and a thermometer.

Computational study of acoustic fields in the SSAW device. To simulate acoustic fields in our cell patterning device, a frequency domain 2D model was developed using COMSOL Multiphysics V5.3a (COMSOL, Stockholm, Sweden). Dimensions of the model were determined with considering those of the experimental device. A perfectly matched layer (PML) domain was set for the underside of the piezoelectric substrate ${ }^{24}$. The piezoelectric substrate domain was modeled by using the 'Solid Mechanics', 'Electrostatics', and 'Piezoelectric Devices' modules. An AC signal was applied to the IDTs on a piezoelectric substrate ${ }^{24}$. The coupling liquid domain was modeled using the 'Pressure Acoustics' module. The solution domain was modeled using the 'Thermoacoustics' module. The bottom and top coverglass domains were modeled using the 'Solid Mechanics' module. The chamber domain was modeled using the 'Pressure Acoustics' module for the PDMS chamber because the shear wave is negligible compared to the longitudinal wave in PDMS $^{24}$. The interface between domains for pressure acoustics and those for solid mechanics was set as the 'Acoustic-solid interaction' boundary. To analyze the effect of reflection at the water/cover interface, the top boundary of the solution domain was set as the 'Impedance' boundary where the acoustic impedance was calculated from the speed of sound and density of the material. Material properties of water, glass, and lithium niobate were obtained from the COMSOL Material Library. Other parameters utilized in the computational study are listed in Supplementary Table 1 and 2.

Acoustic potential $U$ on a single spherical particle, corresponding to a cell in this work, were calculated with Eq. (1):22

$$
U=V_{0}\left[f_{1}\left(\beta_{\mathrm{f}} / 4\right) \operatorname{Re}\left(p \cdot p^{*}\right)-f_{2}\left(3 \rho_{\mathrm{f}} / 8\right) \operatorname{Re}\left(\nu \cdot \nu^{*}\right)\right]
$$

where $f_{1}=1-\left(\beta_{\mathrm{p}} / \beta_{\mathrm{f}}\right)$, and $f_{2}=2\left(\rho_{\mathrm{p}}-\rho_{\mathrm{f}}\right) /\left(2 \rho_{\mathrm{p}}+\rho_{\mathrm{f}}\right)$. $V_{0}$ is the volume of the particle. $\rho_{\mathrm{p}}, \rho_{\mathrm{f}}, \beta_{\mathrm{p}}$, and $\beta_{\mathrm{f}}$ are the density and compressibility of the particle and the fluid, respectively. $p$ and $v$ are the acoustic pressure field and the acoustic velocity field inside the solution calculated from the frequency domain analysis, respectively. $\mathrm{Re}$ indicates the real part of the complex number. The asterisk indicates complex conjugation.

Preparation of the HA-CA hydrogel. HA-CA conjugate was synthesized by modifying HA (MW $200 \mathrm{kDa}$, Lifecore Biomedical, Chaska, MN, USA) with a catechol group using dopamine hydrochloride (Sigma, St. Louis, MO, USA) ${ }^{34,35}$. In brief, HA was dissolved in distilled water at a concentration of $1 \%(w / v)$. Equal molar quantities of 1-ethyl-3-(3-dimethylaminopropyl) carbodiimide (EDC) (TCI Co., Tokyo, Japan) and N-hydroxysulfosuccinimide (NHS) (Sigma) (relative to the HA backbone unit) were then added into the solution and stirred for $30 \mathrm{~min}$. Dopamine hydrochloride was added to the solution at an equal molar ratio to HA, and the $\mathrm{pH}$ of the solution was adjusted to 5.0 using $1 \mathrm{M}$ hydrochloride. The reaction was continued overnight by stirring at room temperature. To eliminate 
unreacted dopamine hydrochloride, the solution was dialyzed against $1 \times$ phosphate buffered saline (PBS) (3 M Korea, Seoul, Korea) at a pH of 5.0 four times each for $6 \mathrm{~h}$ and against distilled water once for $4 \mathrm{~h}$. The resultant solution was then frozen and lyophilized. For gelation, the HA-CA pre-gel solution (dissolved in PBS) was mixed with a sodium periodate solution $\left(\mathrm{NaIO}_{4}\right.$, Sigma) at an equal molar ratio to catechol to oxidize the catechol groups of HA-CA to reactive o-quinones, which forms covalent crosslinks $\mathrm{s}^{34,35}$. The $\mathrm{pH}$ condition of HA-CA hydrogel was adjusted to 7.2-7.4 using $\mathrm{NaOH}$-containing $\mathrm{NaIO}_{4}$ solution for cell culture.

Rheological analysis of the HA-CA hydrogel. The rheological properties of the HA-CA hydrogel with various gel concentrations $(1.5,2$, and $2.5 \%)(\mathrm{w} / \mathrm{v})$ were determined using a rheometer (MCR102, Anton Paar, Graz, Austria). The elastic moduli of the HA-CA hydrogels were measured in frequency sweep mode over $0.1-100 \mathrm{~Hz}$ at a constant shear strain $(2 \%)$. The gelation kinetics (complex viscosity and gelation time) of the HA-CA hydrogels were investigated in time sweep mode at a constant frequency of $1 \mathrm{~Hz}$ and shear strain of $2 \%$. The gelation time of the hydrogels was determined as the crossover point of the storage modulus $\left(G^{\prime}\right)$ and loss modulus $\left(G^{\prime \prime}\right)$.

Indentation test for mouse tissue and hydrogel. Mouse hindlimb muscle tissue and hydrogel samples were dissected by using a biopsy punch with a diameter of $4 \mathrm{~mm}$ and sliced to a thickness of $2 \mathrm{~mm}$. A custom-made indentation instrument ${ }^{61}$ was utilized to measure the mechanical properties of the samples. The indentation tip was a 1-mm-diameter ball of stainless steel, and the indentation speed was $25 \mu \mathrm{m} \mathrm{s}^{-1}$. Indentation forces were recorded at every $2.6 \mu \mathrm{m}$ of indentation depth. The indentation depth was larger than $130 \mu \mathrm{m}$. The contact points were defined by using the 'goodness of fit' method ${ }^{62}$. Briefly, 100 points of data from an arbitrary data point were fitted to the Hertzian contact model ${ }^{63}$ described in equation (2), and the contact point was defined as the data point when the mean-squared error of the fitting is minimized.

$$
F=(4 / 3) \cdot E^{*} R^{0.5} d^{1.5}
$$

where $F, R$, and $d$ are the measured force, the radius of the indentation tip, and the indentation depth, respectively. $E^{*}$ is defined as Eq. (3):

$$
1 / E^{*}=\left(1-\left(\nu_{\text {tip }}\right)^{2}\right) / E_{\text {tip }}+\left(1-\left(\nu_{\text {sample }}\right)^{2}\right) / E_{\text {sample }}
$$

where $E_{\text {tip }}, E_{\text {sample }}$ and $v_{\text {tip }}, v_{\text {sample }}$ are Young's modulus and Poisson's ratio of the indentation tip and sample, respectively. Young's modulus and Poisson's ratio of the tip were $200 \mathrm{GPa}$ and 0.27 , respectively. Poisson's ratio of the hydrogel and tissue sample were both assumed to be $0.45^{63}$.

Preparation of the 3D-aligned cell construct. A double-sided polyimide tape (3M, Maplewood, MN, USA) was used to immobilize the superstrate on the piezoelectric substrate and to create a space for the coupling liquid between the superstrate and the substrate. Two pieces of polyimide tape were attached outside of the SSAW working area to eliminate any interaction with SSAW. The detachable superstrate was placed on the spacers, and coupling liquid was injected into the gap between the SSAW device and the superstrate. Cesium chloride (Samchun Pure Chemical Co., Pyeongtaek, Gyeonggi-do, Korea) solution with a density of $1.9 \mathrm{~g} \mathrm{~cm}^{-3}$ was used as a coupling liquid.

hADSCs (Invitrogen, Carlsbad, CA, USA) were cultured in MesenPRO RS basal medium (Invitrogen) supplemented with MesenPRO RS growth supplement (Invitrogen), 1\% penicillin/streptomycin (Invitrogen), and 1\% GlutaMAX (Invitrogen). HUVECs (Lonza, Basel, Basel-Stadt, Switzerland) were cultured in endothelial cell growth medium-2 (EGM-2).

To prepare the 3D-aligned cell constructs, HUVECs and hADSCs were mixed at ratios of 1:0, 5:1, and 2:1 at a final density of $1 \times 10^{7} \mathrm{cells} \mathrm{ml}^{-1}$ for in vitro study and $1.2 \times 10^{7}$ cells ml ${ }^{-1}$ for in vivo study. The cell mixtures were suspended into the HA-CA pre-gel solution, and $\mathrm{NaIO}_{4}$ solution was added to the cell-laden pregel solution for gelation. Immediately after inducing gelation, the mixtures were loaded into the PDMS chamber on the SSAW device.

The piezoelectric substrate was actuated by the sinusoidal electrical signal at a frequency of $13.928 \mathrm{MHz}$. The SSAW formed by the superposition of two waves with the same magnitude yet opposite directions produced the standing pressure field inside the hydrogel, leading to the patterning of cells. The SSAW was applied for $10 \mathrm{~s}$. The temperature of hydrogel containing cells was maintained below $36^{\circ} \mathrm{C}$ using the cooling system during experiment.

After complete gelation, the cell-laden hydrogel construct with the PDMS chamber was transferred to a culture dish and cultured in a mixture of EGM-2 and fully supplemented MesenPRO RS medium at a ratio of 2:1 for 1 week. For analysis of VEGF secretion, the construct was cultured in VEGF-free medium.

Preparation of hiPSC-EC. The undifferentiated hiPSCs (BJ1) were maintained in $\mathrm{mTeSR}^{\mathrm{TM}} 1$ (STEMCELL Technologies, Vancouver, BC, Canada) on $5 \%$ matrigel at $37^{\circ} \mathrm{C}, 5 \% \mathrm{CO}_{2}$. Differentiation of hiPSCs into ECs was performed following a protocol published by Lee et al. ${ }^{64}$ Briefly, for mesodermal differentiation, enzymatically dissociated clumps of hiPSCs (lower than passage 60) were cultured on
$0.01 \%$ collagen-coated plates in Dulbecco's Modified Eagle's Medium/Nutrient Mixture F-12 (DMEM/F12) medium (Gibco BRL, Gaithersburg, MD, USA) including 20\% Serum Replacement (SR, Gibco BRL) with CHIR99021 $(3 \mu \mathrm{M})$ (STEMCELL Technologies) and fibroblast growth factor 2 (FGF2, $5 \mathrm{ng} \mathrm{ml}^{-1}$ ) (R\&D systems, Minneapolis, MN, USA) for 3 days. The mesodermally differentiated cells were cultured in DMEM/F12 medium including 20\% SR with VEGFA $\left(10 \mathrm{ng} \mathrm{ml}^{-1}\right)$ (R\&D systems), delta-like ligand 4 (DLL4, $25 \mathrm{ng} \mathrm{ml}^{-1}$ ) (R\&D systems), epidermal growth factor (EGF, $5 \mathrm{ng} \mathrm{ml}^{-1}$ ) (R\&D systems), and FGF2 $\left(5 \mathrm{ng} \mathrm{ml}^{-1}\right)$ for another 11 days, and hiPSC-derived CDH5 + cells were purified by magnetic-activated cell sorting (MACS, Miltenyi Biotec, Auburn, CA, USA) ${ }^{64}$.

Viability test. Cytotoxicity was evaluated by staining cells with a live/dead solution (Invitrogen) according to the manufacturer's instructions. Cells stained with green fluorescence for live cells and red fluorescence for dead cells were observed using a confocal microscope (LSM 880, Carl Zeiss, Oberkochen, Baden-Württemberg, Germany). The percentage of viable cells was calculated by counting the live and dead cells in the confocal images.

Immunocytochemistry. Cell-laden hydrogel constructs were fixed with $2 \%$ formaldehyde (Sigma) for $1 \mathrm{~h}$ and then permeabilized with $0.2 \%$ Triton X-100 (Wako, Osaka, Japan) for $30 \mathrm{~min}$ at room temperature. To prevent nonspecific antibody binding, the constructs were incubated with $5 \%$ bovine serum albumin (Wako) for $2 \mathrm{~h}$ at room temperature. The following primary antibodies were applied for $48 \mathrm{~h}$ at $4{ }^{\circ} \mathrm{C}$ : mouse anti-CD31 (Millipore, Schwalbach, Hessen, Germany, Cat\# CBL468, 1:20 dilution), rabbit anti-CD44 (Proteintech, Chicago, IL, USA, Cat\# 15675-1-AP, 1:100 dilution), rabbit anti-VE-cadherin (Cell Signaling Technology, Beverly, MA USA, Cat\# 2500S, 1:100), mouse anti- $\alpha$-SMA (Chemicon, Temecula, CA, USA, Cat\# CBL171, 1:1000 dilution), and rabbit anti-ZO-1 (Invitrogen, Cat\# 61-7300, 1:50 dilution). The constructs were then washed with PBS overnight at $4{ }^{\circ} \mathrm{C}$ and incubated with the following secondary antibodies overnight at $4^{\circ} \mathrm{C}$ : Alexa Fluor 488-conjugated goat anti-mouse IgG (Invitrogen, Cat\# A11001, 1:200 dilution) and Alexa Fluor 594-conjugated goat anti-rabbit IgG (Invitrogen, Cat\# A11002, 1:200 dilution). Nuclei were identified by staining with 4,6-diamidino-2-phenylindole (DAPI; Vector Laboratories, Burlingame, CA, USA) for $30 \mathrm{~min}$ at room temperature. A confocal microscope (LSM 880) was used to observe the stained cells

Image-based quantification. To visualize the spatial distribution of cells in the hydrogel, DiI (Invitrogen)-stained HUVECs or green fluorescent protein (GFP)overexpressing HeLa cells were patterned in $2 \%$ HA-CA hydrogel. The cell density was varied from $1 \times 10^{6} \mathrm{cells} \mathrm{ml}^{-1}$ to $2 \times 10^{7} \mathrm{cells}^{-1}$. For time-lapse imaging, DiI-stained cells at a density of $1 \times 10^{7}$ cells ml ${ }^{-1}$ were patterned in polymerizing $2 \%$ HA-CA hydrogel. Time-lapse images of cells during patterning were obtained by an epifluorescence microscope (Ni-U, Nikon, Tokyo, Japan) with a charge-coupled device (CCD) camera (DS-Q1Mc, Nikon). The blood vessels in mouse hindlimb muscle tissue were visualized via the tail vein injection of $70 \mathrm{kDa}$ FITC-dextran (Sigma) solution. Fluorescence signals of GFP-overexpressing HeLa cells and FITCdextran in blood vessels were imaged using a confocal microscope (LSM 880).

The alignment index (AI) of the blood vessels in tissue samples and the patterned cells in hydrogel samples were obtained by the 2D fast Fourier transform (FFT) approach ${ }^{65}$ using Fiji software ${ }^{66}$ (National Institutes of Health, Bethesda, MD, USA) (Supplementary Fig. 1). The 2D Fourier transforms of fluorescent images of tissues and cells were obtained, and the summation of pixel intensities along a line from the center of the 2D FFT images at an angle from $0^{\circ}$ to $180^{\circ}$ was measured using Fiji software (National Institutes of Health). The peak of the intensity was recentered to $0^{\circ}$, and the ratio of the area of the normalized intensity within $20^{\circ}$ around the peak to the whole area of the intensity was calculated. The $\mathrm{AI}$ of an image was calculated by dividing the area ratio of the image by the ratio of an image with randomly distributed objects $(40 / 180=0.22)$. Higher AI values indicate that the objects in the image are more aligned. The AI value varied from 1 to 4.5 .

(AIP) of the images of patterned cells were cells along the $X, Y$, and $Z$ axes were obtained from the projected images and then normalized to the maximum value of the intensity profile.

To characterize elongated ECs in a quantitative manner, a projection image of $3 \mathrm{D}$ confocal images of CD31-stained cells with $40-\mu \mathrm{m}$ thickness was obtained. Directionality of the ECs was estimated using the 'Directionality' function in Fiji software (National Institutes of Health) for the stacked images. The length of the vessel branch was estimated by converting a stacked image into a skeletonized image and using the 'Analyze Skeleton (2D/3D)' function.

To assess the physical contact between cells, aggregated cells in 3D confocal images were rendered into a single object using the 'Surface' function in Imaris software (Bitplane, Zurich, Switzerland). The volume of the separated objects was visualized by the 'Statistics' function in Imaris MeasurementPro software (Bitplane)

Analysis of paracrine factor secretion. Conditioned medium was collected from each culture dish with cell-laden hydrogel constructs at days $1,3,5$, and 7 . Paracrine factors, including human VEGF, human IL-10 and human PDGF in the collected conditioned medium were detected by enzyme-linked immunosorbent assay (ELISA) kits (R\&D Systems) following the manufacturer's instructions. 
Quantitative real-time polymerase chain reaction (qPCR). To isolate cells from the HA-CA hydrogels, the cell-laden hydrogel constructs were incubated in $1000 \mathrm{U}$ $\mathrm{ml}^{-1}$ of hyaluronidase solution for $20 \mathrm{~min}$ at $37^{\circ} \mathrm{C}$. Degraded hydrogel solution was then centrifuged at $1000 \mathrm{rpm}$ for $5 \mathrm{~min}$ to collect the cell pellet. After decanting the supernatant, the cell pellet was lysed, and total RNA was extracted using a MiniBEST universal RNA extraction kit (TaKaRa, Otsu, Shiga, Japan). RNA samples were reverse-transcribed into cDNA using a PrimeScript ${ }^{\mathrm{TM}}$ strand cDNA synthesis kit (TaKaRa). qPCR was conducted with the synthesized cDNA using a StepOnePlus Real-Time PCR system (Applied Biosystems, Foster City, CA, USA), TaqMan Fast Universal PCR Master Mix (Applied Biosystems), and TaqMan gene expression assays (VE-cadherin (CDH5): Hs00901465_m1, a-SMA (ACTA2): Hs00426835_g1, Tie2 (TEK): Hs00945150_m1 and vWF (VWF): Hs01109446_ml). Target gene expression value was determined by the comparative $C_{\mathrm{t}}$ method and normalized to that of an endogenous housekeeping gene, glyceraldehyde 3-phosphate dehydrogenase (GAPDH: Hs02758991_g1).

Subcutaneous transplantation of the constructs. All animal experiments in the study were reviewed and approved by the Institutional Animal Care and Use Committee (IACUC) at the Yonsei Laboratory Animal Research Center (YLARC; permit number: IACUC-A-201603-172-02). Immediately after fabricating the cellhydrogel constructs in vitro, the constructs were transplanted into subcutaneous pockets in the dorsal regions (two constructs per mouse; right side for the 'Align' group and left side for the 'Random' group) of 6-week-old female Balb/c-nu mice (Orient Bio, Seongnam, Gyeonggi-do, Korea). After a longitudinal incision was made in the mouse dorsal skin, each construct was placed between the skin and muscle layer. The incision was closed with a 6-0 prolene suture (Ethicon, Somerville, NJ, USA). To visualize the vasculatures of the transplanted constructs and the surrounding skin tissue at 1 and 3 weeks post-transplantation, $100 \mu \mathrm{l}$ of $1 \mathrm{mg}$ $\mathrm{ml}^{-1}$ FITC-dextran $(70 \mathrm{kDa}$, Sigma) or fluorescein-isolectin solution (Vector Laboratories) was intravenously injected into the mouse tail vein. The mice were sacrificed $15 \mathrm{~min}$ after injection, and the hydrogel constructs were harvested while attached to skin tissue. The fluorescently labeled vasculatures in the harvested constructs were immediately visualized using a confocal microscope (LSM 880). Constructs with skin tissue were also fixed with $4 \%$ paraformaldehyde (Sigma) overnight at room temperature and embedded in optimal cutting temperature (OCT) compound (CellPath, Newtown, Powys, UK) for histological analysis. After cryosectioning, the slices were stained with $\mathrm{H} \& \mathrm{E}$ and immunofluorescently stained for CD31 (Abcam, Cambridge, Cambridgeshire, UK) to confirm the capillaries in the tissue constructs following standard histological procedures.

\section{Transplantation of the constructs in hindlimb ischemia model. Hindlimb}

ischemia was induced in anesthetized athymic female mice (Balb/c-nu, 6 weeks old, Orient Bio ${ }^{49}$. After skin incision, the left iliac and femoral arteries were permanently ligated using a 6-0 prolene suture (Ethicon). Immediately after ligation of the arteries, 3D cell-hydrogel constructs, pre-matured in vitro for 1 week, were placed onto the defective muscle, fully covering the ischemic region. The mice were divided into four groups: (i) no-treatment, (ii) hydrogel-alone (Gel), (iii) randomly distributed cell-hydrogel construct (Random), and (iv) aligned cell-hydrogel construct (Align). The status of the lower extremity of the left limb on day 28 was evaluated to determine its physiological status score. Amputation of the lower extremity was designated as limb loss, and rotten skin and muscle of the left limb was designated as necrosis. The blood perfusion in ischemic hindlimbs was monitored by serial scanning using a laser Doppler imaging system (MoorLDI2HIR High Resolution, Moor Instruments, Axminster, Devon, UK) on days 0, 2, 7 , 14,21 , and 28 after treatment ${ }^{67}$. Blood flow was quantified using digital colorcoded images from the knee joint region to the toe region, and the perfusion rate was calculated as the ratio of ischemic limb to normal limb. Four weeks after transplantation, the mice were sacrificed for histological analysis.

Histology and immunohistochemistry. Harvested ischemic muscles were fixed with $4 \%$ paraformaldehyde (Sigma) overnight at room temperature and embedded in paraffin for slicing. The tissue slices were stained with H\&E and Masson's trichrome. The fibrotic area was quantified as the percentage of collagen-stained area in Masson's trichrome-stained images using Fiji software (National Institutes of Health). The sectioned specimens were immunofluorescently stained for capillaries and arterioles using anti-CD31 (Abcam, Cat\# ab9498, 1:50 dilution) and antia-SMA antibodies (Santa Cruz Biotechnology, Santa Cruz, CA, USA, Cat\# sc53142 , 1:100 dilution) following standard histological procedures. The densities of capillaries and arterioles were determined by counting CD31-positive capillaries and $\alpha$-SMA-positive microvessels, respectively, and arteriole size was measured using Fiji software (National Institutes of Health) based on the fluorescent images of a-SMA-positive microvessels. The sectioned tissues were also immunofluorescently stained to detect blood vessels formed by transplanted human cells using human-specific anti-CD44 (Abcam, Cat\# ab51037, 1:100 dilution) and antiCD31 (Abcam, Cat\# ab9498, 1:50 dilution). The number of blood vessels consisting of transplanted hADSCs was determined by counting the human CD44-positive vessels in the field of view.
Statistical analysis. All data are presented as the mean \pm standard deviation and were statistically analyzed using GraphPad Prism (GraphPad Software, San Diego, CA, USA). To determine statistical significance, unpaired Student's $t$-tests and oneway or two-way analysis of variance (ANOVA) were used.

\section{Data availability}

The authors declare that all data supporting the findings of this study are available within the paper and its supplementary information file.

Received: 28 April 2018 Accepted: 23 November 2018

Published online: 20 December 2018

\section{References}

1. Khademhosseini, A. \& Langer, R. A decade of progress in tissue engineering. Nat. Protoc. 11, 1775-1781 (2016).

2. Laschke, M. W. \& Menger, M. D. Life is 3D: boosting spheroid function for tissue engineering. Trends Biotechnol. 35, 133-144 (2017).

3. Bajaj, P. et al. 3D biofabrication strategies for tissue engineering and regenerative medicine. Annu. Rev. Biomed. Eng. 16, 247-276 (2014).

4. Datta, P., Ayan, B. \& Ozbolat, I. T. Bioprinting for vascular and vascularized tissue biofabrication. Acta Biomater. 51, 1-20 (2017).

5. Mirabella, T. et al. 3D-printed vascular networks direct therapeutic angiogenesis in ischaemia. Nat. Biomed. Eng. 1, 0083 (2017).

6. Bogorad, M. I. et al. Review: in vitro microvessel models. Lab. Chip. 15, $4242-4255$ (2015)

7. Traore, M. A. \& George, S. C. Tissue engineering the vascular tree. Tissue Eng Part. B Rev. 23, 505-514 (2017).

8. Seifu, D. G., Purnama, A., Mequanint, K. \& Mantovani, D. Small-diameter vascular tissue engineering. Nat. Rev. Cardiol. 10, 410-421 (2013).

9. Stratman, A. N., Malotte, K. M., Mahan, R. D., Davis, M. J. \& Davis, G. E. Pericyte recruitment during vasculogenic tube assembly stimulates endothelial basement membrane matrix formation. Blood 114, 5091-5101 (2009).

10. Baumgartner, I. et al. Constitutive expression of phVEGF $_{165}$ after intramuscular gene transfer promotes collateral vessel development in patients with critical limb ischemia. Circulation 97, 1114-1123 (1998).

11. Annex, B. H. Therapeutic angiogenesis for critical limb ischaemia. Nat. Rev. Cardiol. 10, 387-396 (2013).

12. Claesson-Welsh, L. Vascular permeability-the essentials. Ups J. Med. Sci. 120, 135-143 (2015).

13. Jensen, L., Bangsbo, J. \& Hellsten, Y. Effect of high intensity training on capillarization and presence of angiogenic factors in human skeletal muscle. J. Physiol. 557, 571-582 (2004).

14. Gavin, T. P. et al. Lower capillary density but no difference in VEGF expression in obese vs. lean young skeletal muscle in humans. J. Appl. Physiol. 98, 315-321 (2005)

15. Augustin, H. G. \& Koh, G. Y. Organotypic vasculature: from descriptive heterogeneity to functional pathophysiology. Science 357, eaal2379 (2017).

16. Schütz, K. et al. Three-dimensional plotting of a cell-laden alginate/ methylcellulose blend: towards biofabrication of tissue engineering constructs with clinically relevant dimensions. J. Tissue Eng. Regen. Med. 11, 1574-1587 (2017).

17. Paschos, N. K., Brown, W. E., Eswaramoorthy, R., Hu, J. C. \& Athanasiou, K. A. Advances in tissue engineering through stem cell-based co-culture. J. Tissue Eng. Regen. Med. 9, 488-503 (2015).

18. Dejana, E. Endothelial cell-cell junctions: happy together. Nat. Rev. Mol. Cell Biol. 5, 261-270 (2004)

19. Collins, M. N. \& Birkinshaw, C. Hyaluronic acid based scaffolds for tissue engineering - a review. Carbohydr. Polym. 92, 1262-1279 (2013).

20. Ryu, J. H., Hong, S. \& Lee, H. Bio-inspired adhesive catechol-conjugated chitosan for biomedical applications: a mini review. Acta Biomater. 27, 101-115 (2015).

21. Lee, J., Rhyou, C., Kang, B. \& Lee, H. Continuously phase-modulated standing surface acoustic waves for separation of particles and cells in microfluidic channels containing multiple pressure nodes. J. Phys. D Appl. Phys. 50, 165401 (2017).

22. Guo, F. et al. Controlling cell-cell interactions using surface acoustic waves. Proc. Natl Acad. Sci. USA 112, 43-48 (2015).

23. Naseer, S. M. et al. Surface acoustic waves induced micropatterning of cells in gelatin methacryloyl (GelMA) hydrogels. Biofabrication 9, 015020 (2017).

24. Darinskii, A., Weihnacht, M. \& Schmidt, H. Computation of the pressure field generated by surface acoustic waves in microchannels. Lab. Chip. 16, 2701-2709 (2016)

25. Chen, K. et al. Rapid formation of size-controllable multicellular spheroids via 3D acoustic tweezers. Lab. Chip. 16, 2636-2643 (2016). 
26. Guo, F. et al. Reusable acoustic tweezers for disposable devices. Lab. Chip. 15, 4517-4523 (2015).

27. Traktuev, D. O. et al. Robust functional vascular network formation in vivo by cooperation of adipose progenitor and endothelial cells. Circ. Res. 104, 1410-1420 (2009).

28. Rehman, J. et al. Secretion of angiogenic and antiapoptotic factors by human adipose stromal cells. Circulation 109, 1292-1298 (2004).

29. Merfeld-Clauss, S. et al. Adipose stromal cells differentiate along a smooth muscle lineage pathway upon endothelial cell contact via induction of activin A. Circ. Res. 115, 800-809 (2014).

30. Mendel, T. A. et al. Pericytes derived from adipose-derived stem cells protect against retinal vasculopathy. PLoS ONE 8, e65691 (2013).

31. Karantalis, V. \& Hare, J. M. Use of mesenchymal stem cells for therapy of cardiac disease. Circ. Res. 116, 1413-1430 (2015).

32. Liang, Y., Walczak, P. \& Bulte, J. W. The survival of engrafted neural stem cells within hyaluronic acid hydrogels. Biomaterials 34, 5521-5529 (2013).

33. Bian, L., Guvendiren, M., Mauck, R. L. \& Burdick, J. A. Hydrogels that mimic developmentally relevant matrix and N-cadherin interactions enhance MSC chondrogenesis. Proc. Natl Acad. Sci. USA 110, 10117-10122 (2013).

34. Park, H. J. et al. Catechol-functionalized hyaluronic acid hydrogels enhance angiogenesis and osteogenesis of human adipose-derived stem cells in critical tissue defects. Biomacromolecules 17, 1939-1948 (2016).

35. Shin, J. et al. Tissue adhesive catechol-modified hyaluronic acid hydrogel for effective, minimally invasive cell therapy. Adv. Funct. Mater. 25, 3814-3824 (2015).

36. Connacher, W. et al. Micro/nano acoustofluidics: materials, phenomena, design, devices, and applications. Lab. Chip. 18, 1952-1996 (2018).

37. Bouyer, C. et al. A bio-acoustic levitational (BAL) assembly method for engineering of multilayered, $3 \mathrm{D}$ brain-like constructs, using human embryonic stem cell derived neuro-progenitors. Adv. Mater. 28, 161-167 (2016).

38. Wiklund, M. Acoustofluidics 12: biocompatibility and cell viability in microfluidic acoustic resonators. Lab. Chip. 12, 2018-2028 (2012).

39. Bergers, G. \& Song, S. The role of pericytes in blood-vessel formation and maintenance. Neuro. Oncol. 7, 452-464 (2005).

40. Carmeliet, P. et al. Targeted deficiency or cytosolic truncation of the VEcadherin gene in mice impairs VEGF-mediated endothelial survival and angiogenesis. Cell 98, 147-157 (1999).

41. Risau, W. Mechanisms of angiogenesis. Nature 386, 671-674 (1997).

42. Crisan, M. et al. A perivascular origin for mesenchymal stem cells in multiple human organs. Cell. Stem Cell. 3, 301-313 (2008).

43. Cao, R. et al. Angiogenic synergism, vascular stability and improvement of hind-limb ischemia by a combination of PDGF-BB and FGF-2. Nat. Med. 9, 604-613 (2003).

44. Nakagami, H. et al. Novel autologous cell therapy in ischemic limb disease through growth factor secretion by cultured adipose tissue-derived stromal cells. Arterioscler. Thromb. Vasc. Biol. 25, 2542-2547 (2005).

45. Nauta, A. J. \& Fibbe, W. E. Immunomodulatory properties of mesenchymal stromal cells. Blood 110, 3499-3506 (2007).

46. Holnthoner, W. et al. Adipose-derived stem cells induce vascular tube formation of outgrowth endothelial cells in a fibrin matrix. J. Tissue Eng. Regen. Med. 9, 127-136 (2015).

47. Li, S. et al. Hydrogels with precisely controlled integrin activation dictate vascular patterning and permeability. Nat. Mater. 16, 953-961 (2017).

48. Rich, M. H. et al. Material-mediated proangiogenic factor release pattern modulates quality of regenerated blood vessels. J. Control Release 196, 363-369 (2014).

49. Niiyama, H., Huang, N. F., Rollins, M. D. \& Cooke, J. P. Murine model of hindlimb ischemia. J. Vis. Exp. 23, 1035 (2009).

50. Silva, G. T. \& Bruus, H. Acoustic interaction forces between small particles in an ideal fluid. Phys. Rev. E 90, 063007 (2014).

51. Richards, D., Jia, J., Yost, M., Markwald, R. \& Mei, Y. 3D Bioprinting for Vascularized Tissue Fabrication. Ann. Biomed. Eng. 45, 132-147 (2017).

52. Ding, X. et al. On-chip manipulation of single microparticles, cells, and organisms using surface acoustic waves. Proc. Natl Acad. Sci. USA 109, 11105-11109 (2012).

53. Parikh, P. P., Liu, Z.-J. \& Velazquez, O. C. A molecular and clinical review of stem cell therapy in critical limb ischemia. Stem Cells Int. 2017, 3750829 (2017).

54. Chang, W. G. \& Niklason, L. E. A short discourse on vascular tissue engineering. NPJ Regen. Med. 2, 7 (2017).

55. Gao, G. et al. Tissue engineered bio-blood-vessels constructed using a tissuespecific bioink and 3D coaxial cell printing technique: a novel therapy for ischemic disease. Adv. Funct. Mater. 27, 1700798 (2017).

56. Cui, L., Ju, Y., Ding, L., Trejo-Morales, M. \& Olfert, I. M. Arteriolar and venular capillary distribution in skeletal muscles of old rats. J. Gerontol. A. Biol. Sci. Med. Sci. 63, 928-935 (2008).
57. Kassab, G. S. Scaling laws of vascular trees: of form and function. Am. J. Physiol. Heart Circ. Physiol. 290, H894-H903 (2006).

58. Aird, W. C. Endothelial cell heterogeneity. Cold Spring Harb. Perspect. Med. 2, a006429 (2012).

59. Schaupper, M., Jeltsch, M., Rohringer, S., Redl, H. \& Holnthoner, W. Lymphatic vessels in regenerative medicine and tissue engineering. Tissue Eng. Part. B Rev. 22, 395-407 (2016).

60. Subramanian, H., Varadan, V. K., Varadan, V. V. \& Vellekoop, M. J. Design and fabrication of wireless remotely readable MEMS based microaccelerometers. Smart Mater. Struct. 6, 730-738 (1997).

61. Cho, S. H. et al. Micropatterned pyramidal ionic gels for sensing broad-range pressures with high sensitivity. ACS Appl. Mater. Interfaces 9, 10128-10135 (2017).

62. Gavara, N. Combined strategies for optimal detection of the contact point in AFM force-indentation curves obtained on thin samples and adherent cells. Sci. Rep. 6, 21267 (2016).

63. Flores-Merino, M. V. et al. Nanoscopic mechanical anisotropy in hydrogel surfaces. Soft Matter 6, 4466-4470 (2010).

64. Lee, S.-J. et al. Enhanced therapeutic and long-term dynamic vascularization effects of human pluripotent stem cell-derived endothelial cells encapsulated in a nanomatrix gel. Circulation 136, 1939-1954 (2017).

65. Ayres, C. E. et al. Measuring fiber alignment in electrospun scaffolds: a user's guide to the 2D fast Fourier transform approach. J. Biomater. Sci. Polym. Ed. 19, 603-621 (2008).

66. Schindelin, J. et al. Fiji: an open-source platform for biological-image analysis. Nat. Methods 9, 676-682 (2012).

67. Cho, S. W. et al. Improvement of postnatal neovascularization by human embryonic stem cell-derived endothelial-like cell transplantation in a mouse model of hindlimb ischemia. Circulation 116, 2409-2419 (2007).

\section{Acknowledgements}

This work was supported by the National Research Foundation of Korea (NRF) grants funded by the Korea Government (2015R1A2A2A01007602, 2017R1A2B3005994, 2018R1A2A3075287), the Institute of Convergence Science (ICONS) at Yonsei University, the Institute for Basic Science (IBS-R026-D1), Global Research Cooperation Funding provided by Emory University and Yonsei University, and Brain Korea 21 Plus Project (Mechanical Technology Global Leader Program for Society Development, Initiative for Biological Function \& Systems) in 2018.

\section{Author contributions}

B.K., J.S., S.-W.C., and H.L. designed the research project. B.K. and C.R. prepared the cell patterning instrument. B.K. conducted image-based and numerical analyses. B.K. and J.S. fabricated the 3D-aligned cell construct. B.K. and J.S. performed the in vitro experiments. J.S. and H.J.-P. performed the animal experiments. D.K. performed the indentation test. S.J.-L. and Y.S.-y. prepared iPSC-EC. B.K., J.S., S.-W.C., and H.L. wrote the manuscript.

\section{Additional information}

Supplementary Information accompanies this paper at https://doi.org/10.1038/s41467018-07823-5.

Competing interests: The authors declare no competing interests.

Reprints and permission information is available online at http://npg.nature.com/ reprintsandpermissions/

Journal peer review information: Nature Communications thanks the anonymous reviewers for their contributions to the peer review of this work.

Publisher's note: Springer Nature remains neutral with regard to jurisdictional claims in published maps and institutional affiliations.

Open Access This article is licensed under a Creative Commons Attribution 4.0 International License, which permits use, sharing, adaptation, distribution and reproduction in any medium or format, as long as you give appropriate credit to the original author(s) and the source, provide a link to the Creative Commons license, and indicate if changes were made. The images or other third party material in this article are included in the article's Creative Commons license, unless indicated otherwise in a credit line to the material. If material is not included in the article's Creative Commons license and your intended use is not permitted by statutory regulation or exceeds the permitted use, you will need to obtain permission directly from the copyright holder. To view a copy of this license, visit http://creativecommons.org/ licenses/by/4.0/.

(C) The Author(s) 2018 